(c) 2016 IEEE. Personal use of this material is permitted. Permission from IEEE must be obtained for all other users, including reprinting/ republishing this material for advertising or promotional purposes, creating new collective works for resale or redistribution to servers or lists, or reuse of any copyrighted components of this work in other works. 


\title{
An UDWDM-PON using low-cost coherent transceivers with limited tunability and heuristic DWA
}

\author{
Vicent Sales, Josep Segarra, Víctor Polo, J. Camilo Velásquez and Josep Prat
}

\begin{abstract}
A new Passive Optical Network (PON) for access, making use of Ultra Dense Wavelength Division Multiplexing (UDWDM) by densely spacing channels at few $\mathrm{GHz}$, and introducing the "wavelength-to-the-user" concept, is proposed. The key challenge will be developing low-cost coherent transceivers, providing an excellent selectivity while avoiding filters, and furnishing high sensitivity, which will allow high splitting ratios, large number of users and long distance reach. The Optical Distribution Network (ODN) at the outside plant is based on splitters and kept compatible with legacy systems. Optical Network Unit (ONU) designs realized with coherent transceivers using one or two lasers are presented and the corresponding Optical Line Terminal (OLT) architectures are introduced. The ONUs at customer premises own lasers with limited thermal tunability and their wavelengths are randomly distributed in a band. By using heuristic Dynamic Wavelength Assignment (DWA) schemes and extending the original working band, the required optical band is obtained and optimized. In activation processes, ONU acceptances up to $99.9 \%$ are achieved. Furthermore, in operation scenario under indoors and also under outdoors environmental conditions, ONU blocking probabilities below $0.1 \%$ and ONU availability ratios (OARs) up to $99.9 \%$ are demonstrated. The PON is dimensioned according to the number of deployed users and system reach; moreover, power safety and also fiber nonlinearities constraints are evaluated, illustrating the characteristics of the projected network. Finally, the coexistence with legacy networks is discussed.
\end{abstract}

Index Terms-Fiber-To-The-Home (FTTH); Passive Optical Network (PON); Optical Network Unit (ONU), Optical Line Terminal (OLT); coherent transceiver; Ultra Dense Wavelength Division Multiplexing (UDWDM); Dynamic Wavelength Assignment (DWA).

Manuscript received March 11, 2016.

This work was supported in part by the European COCONUT Project (G.A. 318515) and by the Spanish Ministry of Science and Innovation Project FLIPER, under grant TEC2015-70835-R.

The authors are with the Universitat Politècnica de Catalunya (UPC), Signal Theory and Communications Department (TSC), Barcelona, E-08034, Spain (e-mail: vicent@tsc.upc.edu).

\section{INTRODUCTION}

$\mathbf{P}$ resently, GPON and EPON standards based on Time Division Multiplexing (TDM) are the developed FTTH technologies, being extensively deployed with a fiber and split-built Optical Distribution Network (ODN) (Fig. 1); however they employ only two wavelengths, thus making poor use of spectral efficiency in optical fibers, but on the other hand core networks are reaching now its limits. Their TDM evolutions to furnish higher bit rates are XG-PON [1] and 10GE-PON [2], respectively, but with increased power consumption and low economic efficiency per customer. To provide higher network capacity, several schemes have been introduced, including WDM [3], hybrid WDM/TDM [4], Orthogonal Frequency Division Multiplexing (OFDM) [5] and, finally, Time and Wavelength Division Multiplexing (TWDM) [6]; however, low-cost and backwards compatibility requirements are not yet fulfilled. The next generation NGPON2 recommendation [7] is based on TWDM by a sort of stacking from 4 to 8 XG-PONs and defining an optional set of Point-to-Point (PtP) wavelengths (WDM-PON), providing the coexistence with legacy systems through wavelength overlay and keeping the split-based ODN, while still using the Intensity Modulation and Direct Detection (IM/DD) transmission format. NG-PON2 has three key elements: a Wavelength Multiplexer (WM) at the Optical Line Terminal (OLT) [8], a Coexistence Element (CE) at the ODN for coexistence with legacy PONs, and a tunable filter at the Optical Network Unit (ONU). Severe requirements are claimed for channel isolation, demanding the use of cascaded filters or increased channel separation; and also fast filter tuning time, limiting considerably the technology choices, while increasing the implementation costs [9].

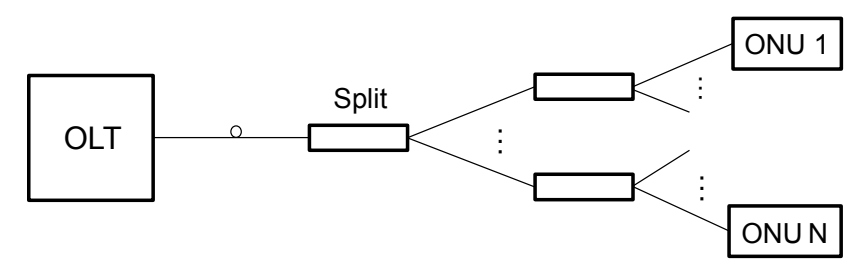

Fig. 1. PON with OLT at central office and ODN based on splitters.

Alternatively, the proposal of a coherent Ultra Dense WDM (UDWDM) permits realizing a novel Wavelength-ToThe-User (WTTU) approach, furnishing each customer a 
dedicated wavelength while spacing channels at a few $\mathrm{GHz}$ [10], and besides the split-based ODN can be maintained. According to ITU recommendation [11], the grid will evolve to finer $12.5 \mathrm{GHz}$ slots, so next step will be down to 6.25 $\mathrm{GHz}$, allowing a scalable PON for high number of users [12].

Furthermore, employing coherent detection provides very good features: 1) excellent wavelength selectivity, avoiding optical filters; 2) high sensitivity, enabling long reach and high splitting ratios and 3) high speed WTTU connections; and additionally, a digital signal processing leverages equalization and forward error correction (FEC).

With this purpose, an UDWDM-PON is developed under the EU FP7 COst-effective COhereNt Ultra-dense-WDMPON for lambda-To-the-user access (COCONUT) project [13-14], as a seamless evolution from present PON deployments in order to support new emerging customer applications and also to serve mobile front-haul and backhaul applications. By using coherent techniques a higher power budget $(>45 \mathrm{~dB})$ is reached thanks to enhanced sensitivity $(<-50 \mathrm{dBm})$, allowing to serve higher number of users (up to 256) and long distances (up to $60 \mathrm{~km}$ ), being each user served with flexible data rates from $1.25 \mathrm{~Gb} / \mathrm{s}$ up to $10 \mathrm{~Gb} / \mathrm{s}$ to provide different services.

When realizing an UDWDM-PON, for each working user, a tunable laser (TL) is needed for transmission and another TL as local oscillator (LO) for coherent reception, and normally two different wavelengths are used for down and up transmission to avoid Rayleigh Backscattering (RB). The tuning range of the lasers is a key parameter for achieving a low-cost implementation of the ONU, leading to choose single-mode single-electrode semiconductor lasers, like distribute feedback (DFB), but with limited tunability of about $0.1 \mathrm{~nm} /{ }^{\circ} \mathrm{C}$ by heating and/or cooling control [15-16]. Using ECL-lasers in coherent detection [17] and DFB-lasers in direct modulation schemes has been shown [18]. Manufacturing DFB-lasers yields random wavelengths with statistical exponential distribution over few nanometers range [15]; but this range can be extended to a larger optical band, of e.g. 10 to $40 \mathrm{~nm}$, simply by merging lasers with different nominal wavelengths in a number of sub-bands and, hereafter, the random wavelengths have approximately a uniform distribution in a band (Fig. 2). When the ONU lasers sources exhibit random wavelengths distributed in a band with limited thermal tunability, which is used to allocate wavelengths avoiding collisions, we have the Statistical UDWDM (SUDWDM) approach; hence, to efficiently distribute the spectrum in activation and also during operation, some Dynamic Wavelength Assignments (DWAs) algorithms schemes were introduced [19].

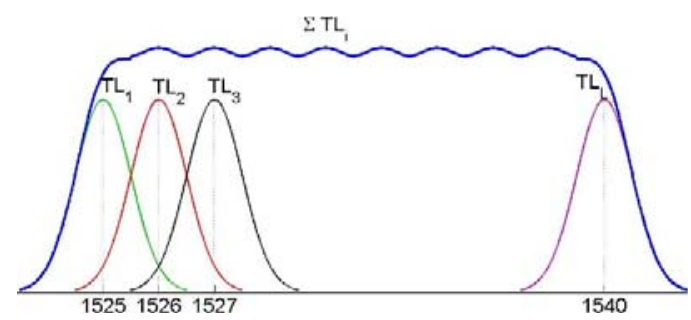

Fig. 2. Approximation of a random wavelength uniform distribution in a band by merging lasers of different nominal wavelengths.
In this work we present the design of the COCONUT UDWDM-PON, by furnishing a WTTU approach and also introducing the proposed architectures for the ONUs at users' premises and for the OLT at the Central Office (CO), where to cope with the required full optical band, we characterize the WMs employed in the OLT realization. DWA schemes in activation and operation processes are proposed, simulated, improved and evaluated to calculate the needed optical bandwidth. Afterwards, the whole network is dimensioned for the presented ONU, OLT and ODN architectures, different number of users and long reach; furthermore, the power safety and the nonlinearities are considered for safe and correct operation, respectively. At last, the coexistence with legacy systems is projected.

This article is organized in the following sections: section II presents ONU and OLT architectures and the modulation techniques, in section III some DWA paradigms and schemes are introduced and evaluated, in section IV a dimensioning of the UDWDM-PON is proposed and its characteristics are shown, section $\mathrm{V}$ tackles the wavelength planning and the coexistence with legacy systems, and, finally, in section VI the conclusions are discussed.

\section{Designing ONU AND OLT ARChITECTURES}

The proposed architectures for the network elements are based on coherent transceivers. At the ONU side the most important goal is to have a low-cost realization in order to provide an affordable deployment, which is achieved by using TLs based on cheap DFB-lasers. At the CO side, the OLT implementation will reproduce the same ONU design for each user, integrating all counterparts in full unique OLT equipment covering all deployed ONUs of the PON.

\section{A. ONU architectures}

Firstly, we introduce ONU designs based on coherent homodyne detection, using a TL as LO for reception and another TL as transmitter, with external or direct modulation, and employing two different wavelengths in separated bands for up and down transmission to avoid RB crosstalk. The TLs are non-preselected with nominal wavelengths uniformly distributed in each band, and of lowcost DFB type with limited tunability adjusted by heating. DFB-lasers exhibit typically a constant linear variation with temperature of $0.1 \mathrm{~nm} /{ }^{\circ} \mathrm{C}$ approximately between 20 to $50^{\circ} \mathrm{C}$ [19], covering $3 \mathrm{~nm}$, thus enabling to tune up to 60 channels spaced at $6.25 \mathrm{GHz}$ in $\mathrm{C}$-band. This temperature range is easily reached with a Peltier, and also even just by heating applying an offset. With different bands or with the same band for up and down, a coupler separates both directions.

In the external modulation case (Fig. 3, a), an ASK or PSK modulation is obtained with a Mach-Zehnder (MZ) modulator controlled by the input data at $1.25 \mathrm{~Gb} / \mathrm{s}$ bit rate [20]. The detection is enabled by an optical hybrid, which can be $90^{\circ}$ or $120^{\circ}$, and 3 or 4 PIN photodiodes with integrated transimpedance amplifier (TIA). In ASK format, an independent polarization simplified receiver can be realized with a polarization beam splitter (PBS) [21].

When using direct modulation (Fig. 3, b), the modulation is done varying the input laser current by the input data; there, the typical employed coherent modulation is DPSK. 
(a)
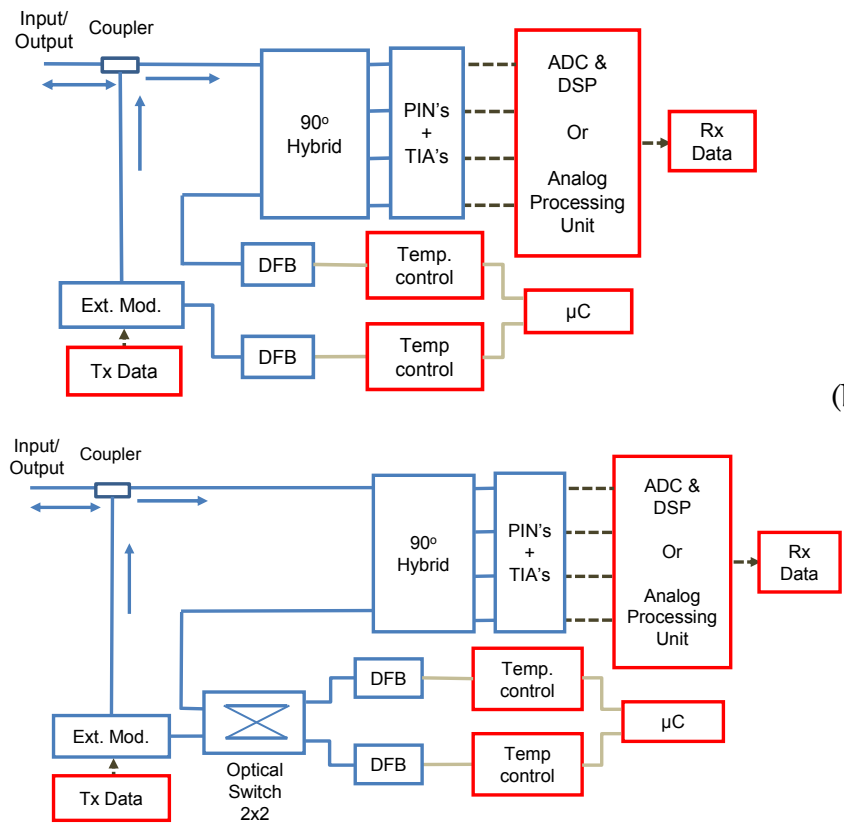

(c)

(d)

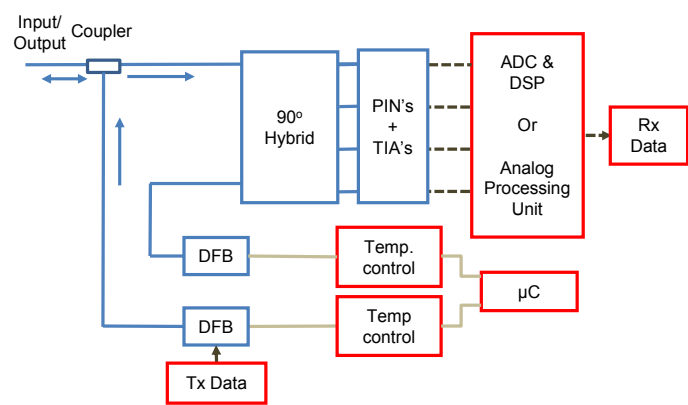

(b)

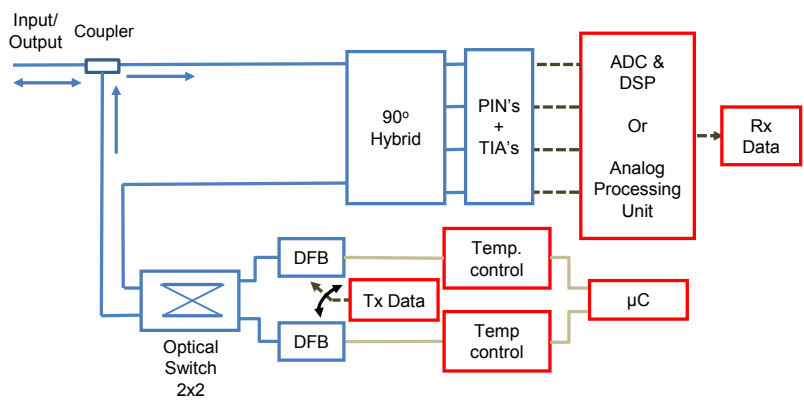

Fig. 3. ONU transceiver block diagrams with coherent homodyne detection. Using two TLs in two different bands: (a) with external ASK or PSK modulation, (b) with direct DPSK modulation. Using two TLs sharing the same optical band: (c) with external ASK or PSK modulation, (d) with direct DPSK modulation.

A thermally tuned directly phase modulated DFB-laser at the ONU transmitting in DPSK at $1.25 \mathrm{~Gb} / \mathrm{s}$, with a coherent homodyne receiver at the OLT showing a BER below the FEC margin of $10^{-3}$ up to $60^{\circ} \mathrm{C}$, has been demonstrated [18]. This furnishes the simplest coherent architecture providing the lowest cost, because it avoids an expensive MZ modulator. Furthermore, recently, to flexibly adapt to different bit rates, transmissions from $1.25 \mathrm{~Gb} / \mathrm{s}$ to $5 \mathrm{~Gb} / \mathrm{s}$ with direct modulated DFBs have been shown [22], operating with either DPSK or DQPSK formats and maintaining the $6.25 \mathrm{GHz}$ channelization. The transmission is realized with two or four level equalized signal, while the reception is the same for both formats and only the precoder is modified, a process that can be done by software.

When using the same sole band for up/down, again both TLs have different wavelengths uniformly distributed; or the same statistics but with a fixed wavelength difference between TLs, paired in a selection procedure for an easier activation process. External and direct modulations are also considered and each TL can act as LO or as transmitter; this is selected by a $2 \times 2$ Optical Switch (OS), which can also simplify the activation process (Fig. 3, c and d) [23-24]. The OS can be of micro-electro-mechanical systems (MEMS) type, which is very cheap but has a switching time of some ms. However, if a Half-Duplex (HD) transmission mode is used [23], employing only one operating TL and the same wavelength for up/down, a switching time in the order of ns is needed. This can be achieved by using an OS of LiNbO3 technology, although it is an expensive solution, but then only one TL can be employed, and the other TL if present can be for back-up. The OS can be avoided in a simple and low-cost design by passing the connections directly, then the TLs are fixed for reception and transmission, and thus the TLs cannot interchange their functionality.
Additionally, a DPSK downstream coherent heterodyne detection at the ONU and upstream at the OLT can be similarly implemented with two PDs. The upstream DPSK modulation at the ONU can be realized by introducing data to a cost-effective Reflective Semiconductor Optical Amplifier (RSOA) transmitting in DPSK at $1.25 \mathrm{~Gb} / \mathrm{s}$ (Fig. 4) [25]. In this way, a unique DFB-laser can be used at the ONU, combining both functions as detection with LO and up-transmission, where a circulator isolates the TL and the RSOA to prevent reflections, although a simple splitter can realize the same isolation function with similar results. Hence, up and down signal spectrums do not overlap and, therefore, RB disturbance is avoided. Nevertheless, the occupied wavelength channel by each ONU is double the width employed by each single TL when using two TLs in separated bands or in the same band.

Besides, the residual intensity modulation (IM) effect in direct phase modulation was eased integrating a DFB and an electro-absorption modulator (EAM) (DEML), which was tested for homodyne and heterodyne detections [26].

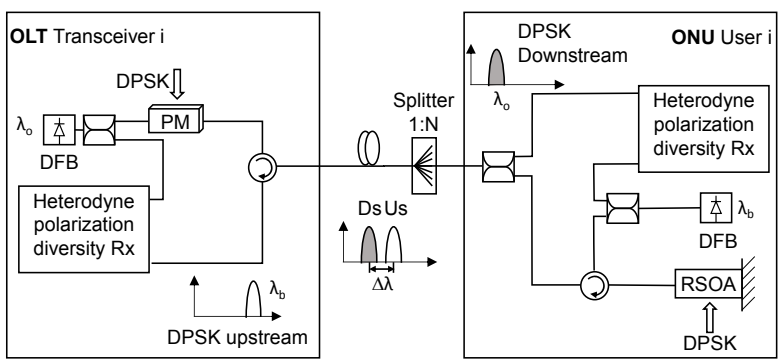

Fig. 4. OLT and ONU transceiver block diagrams with heterodyne coherent detection. At the ONU, an RSOA is a DPSK modulator and only one DFB is used. Down/up spectrums do not overlap. 
Finally, a dual output DEML monolithically integrating a DFB and an EAM in a chip has been demonstrated, with ASK modulation format at the ONU, where the DFB is used for both transmitter and LO [27]. A $2.5 \mathrm{~Gb} / \mathrm{s}$ full-duplex transmission with heterodyne detection has been tested, modulating in ASK for upstream and in DPSK for downstream. The receivers at the ONU and OLT use only one PD per polarization, achieving a high sensitivity. The frequency offset between the signal and the LO is $5 \mathrm{GHz}$, thus enabling a channel spacing of $12.5 \mathrm{GHz}$ between users.

\section{B. OLT architectures}

The OLT implementation is likewise based on coherent transceivers, serving the whole deployed ONUs in the PON. A very simple architecture is realized with power splitters, covering the whole deployed working optical bands without any filtering, whenever using different or the same band for upstream and downstream (Fig. 5, a). A circulator separates up and down transmission in two branches, and at each branch an optical amplifier (OA), which can be an Erbium Doped Fiber Amplifier (EDFA) or a Semiconductor Optical Amplifier (SOA), is introduced to satisfy the power budget.

The OLT owns an Optical Spectrum Analyzer (OSA), inserted in the PON trunk through an asymmetric coupler, monitoring continuously all wavelengths present in the PON (Fig. 5). The OLT will control that all wavelengths are correct in power and allocation, during activation processes and in operation; making decisions about reassignments, if needed, to deal with temperature conditions, by heuristic DWAs schemes [19] and employing OLT-ONU messages in the Medium Access Control (MAC) layer.

We present another OLT architecture based on a $1 \mathrm{xM}$ ports Arrayed Waveguide Grating (AWG) as WM, combined with splitters to serve a high number of users (Fig. 5, b); thus, providing lower Insertion Loss (IL) and then better power budget, and even avoiding amplification for moderate distance reach because of the high coherent detection sensitivity. However, the AWG spectral efficiency, which is the ratio between the $3 \mathrm{~dB}$ response and the channel spacing, is only of $75 \%$ for a $3 \mathrm{~dB}$ flat-top bandwidth and, hereafter, some channels will suffer high attenuation because of low response of the AWG in the transition bands.
In order to have full spectral $100 \%$ efficiency, we propose to use an athermal AWG combined with a thermal AWG or two thermal AWGs, by tuning the spectral response interleaving the output channels using the temperature control in such a way that, when a maximum is achieved in one AWG-port, in the other neighbor AWG-port there is a minimum and vice-versa. In this way, both AWGs have complementary frequency responses and, therefore, a $100 \%$ spectral efficiency is achieved (Fig. 5, b). If down and up transmissions are done in different bands, half of the Mports of each AWG can be devoted to the down-band and the other half to the up-band. If down and up transmissions share the same band, each port will serve both directions. If there are not enough ports in an AWG to cover both down/up-bands, the same architecture can be duplicated: one branch with two AWGs can be used for down and the other branch with two more AWGs for up, with a circulator separating down and up branches.

Both proposed OLT architectures fit all the ONU designs presented previously by fulfilling at the OLT side the twin transmission and reception counterparts, implementing the particular selected homodyne or heterodyne detection.

To cover the whole operation band with limited thermally tuned DFB-lasers for transmission and reception, the band is divided in sub-bands, where each sub-band will have DFB-lasers with nominal wavelength in the center of the sub-band and, therefore, the complete band is covered tuning the DFB-lasers. Hence, a similar band distribution in sub-bands used to achieve a wavelength uniform distribution for the ONUs can be used at the OLT implementation for tuning purposes. Then, the required TLs organized in sub-bands are distributed for transmission and reception at the ports of the OLT, which can be done in both proposed OLT designs (Fig. 5).

\section{AWG characterization}

Several athermal and thermal AWGs are measured, characterizing their IL, spectral response and tuning range in order to evaluate their use in the OLT design.

Two sorts of AWGs are available: gaussian passband and flat-top passaband, presenting a gaussian or a flat-top passband channel shape, respectively.
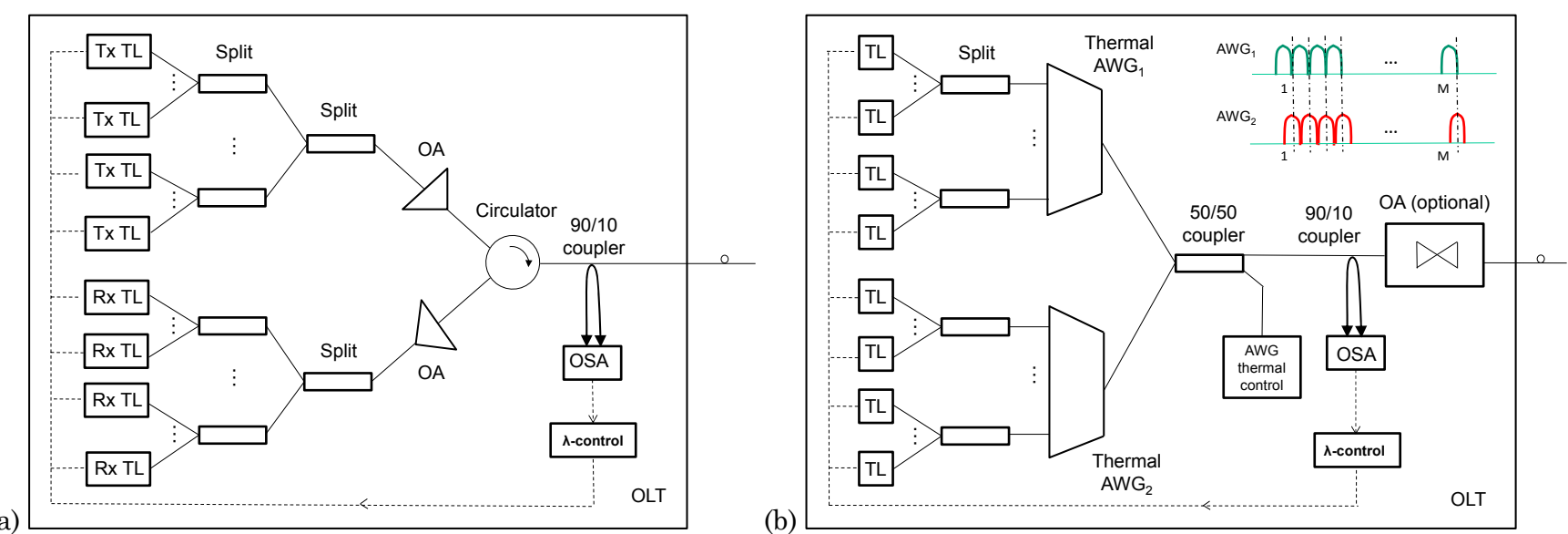

Fig. 5. (a) OLT architecture realized with splitters covering full optical band/s. (b) OLT block diagram based on splitters and two thermal AWGs with complimentary frequency response providing $100 \%$ spectral efficiency. 
In a first setup scenario, a single $100 \mathrm{GHz}$ channel spaced athermal gaussian 41-port $\mathrm{AWG}_{1}$ was tested to evaluate its spectral response (Fig. 6). An EDFA was used as a broadband light generator and was directly connected to one input of a $2 \times 2$ optical coupler, whose output ports were connected to two AWGs. Two output $\mathrm{AWG}_{1}$-ports were connected to an OSA through a $3 \times 3$ coupler and two OSs, which select the output port to be measured. The Amplified Spontaneous Emission (ASE) response of the EDFA was compensated to provide an exact AWG spectral response.

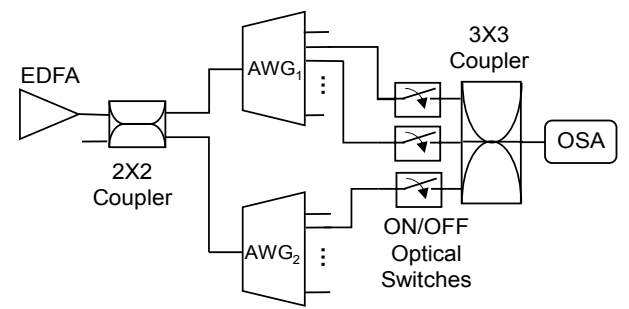

Fig. 6. Measurement setup: single and tuning AWG scenarios.

The ITU channels $32(1551.72 \mathrm{~nm})$ and $33(1550.92 \mathrm{~nm})$, spaced $100 \mathrm{GHz}$, were filtered through the AWG and the Full Width Half Maximum (FWHM) or $3 \mathrm{~dB}$ bandwidth obtained was $60 \mathrm{GHz}$, providing a $60 \%$ spectral efficiency. The IL was in the order of $3.0 \mathrm{~dB}$ and the crosspoint (CP) between these consecutive channels was around $11 \mathrm{~dB}$ below IL, showing a maximum IL (MIL) of $14 \mathrm{~dB}$ (Fig. 7).

In the same scenario, a $100 \mathrm{GHz}$ thermal flat-top 40-port AWG without temperature control was also tested for ITU channels $29(1554.13 \mathrm{~nm})$ and $30(1553.33 \mathrm{~nm})$, providing an IL in the order of $7.5 \mathrm{~dB}$ and showing a $77.5 \mathrm{GHz}$ FWHM, so around $75 \%$ spectral efficiency (Fig. 8). The CP was around $6.4 \mathrm{~dB}$ below the IL, thus the MIL was $14 \mathrm{~dB}$.

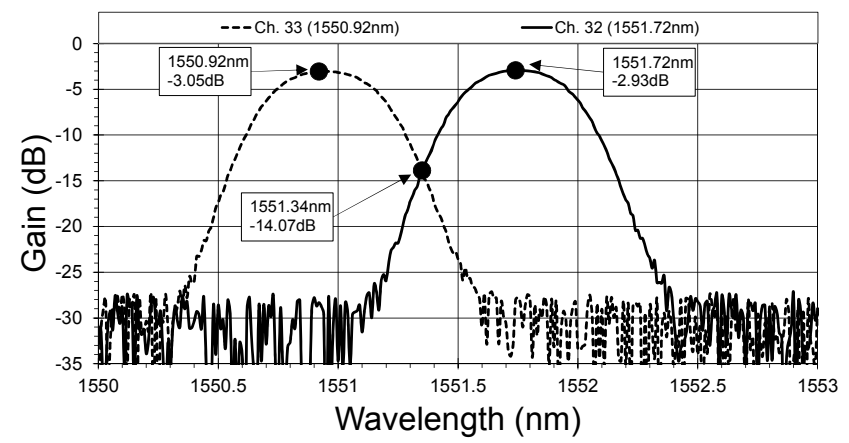

Fig. 7. Athermal gaussian $100 \mathrm{GHz}$ AWG response.

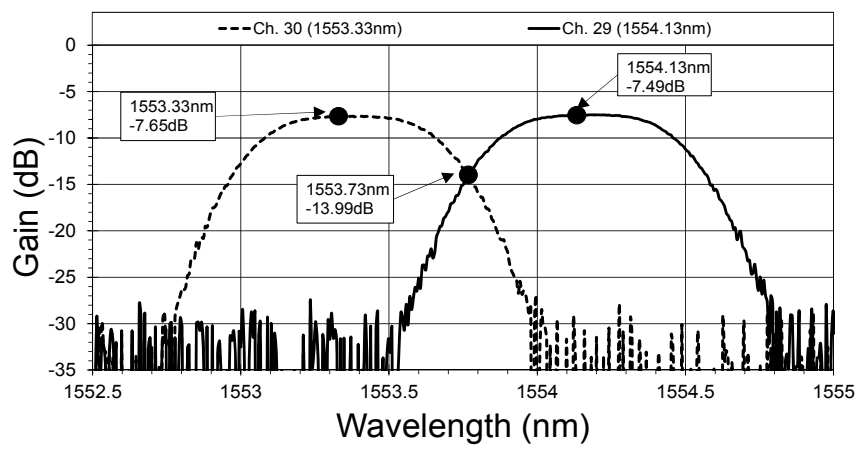

Fig. 8. Thermal flat-top $100 \mathrm{GHz}$ AWG response.
Afterward, in a second tuning setup scenario (Fig. 6), two AWGs were measured simultaneously to test the thermal performance (Fig. 9). A third OS connects the signal from the thermal $\mathrm{AWG}_{2}$, which is controlled by temperature, to the OSA, tuning the $\mathrm{AWG}_{2}$ channel $50 \mathrm{GHz}$ from the nominal frequency in order to allocate its peak at the $\mathrm{CP}$ of the other two channels of $\mathrm{AWG}_{1}$, with all OSs ON. Once the peak is centered, the channels are measured separately.

The peak of channel 33 in thermal gaussian $\mathrm{AWG}_{2}$ was tuned to be allocated at the $\mathrm{CP}$ of channels 32 and 33 of $\mathrm{AWG}_{1}$, which is kept without temperature variation. Once the temperature in $\mathrm{AWG}_{2}$ is increased up to $60^{\circ} \mathrm{C}$, which is the maximum operative rating, the peak reached the CP. Figs. 9 and 10 show the interleaving of AWG channels for gaussian and flat-top responses, respectively.

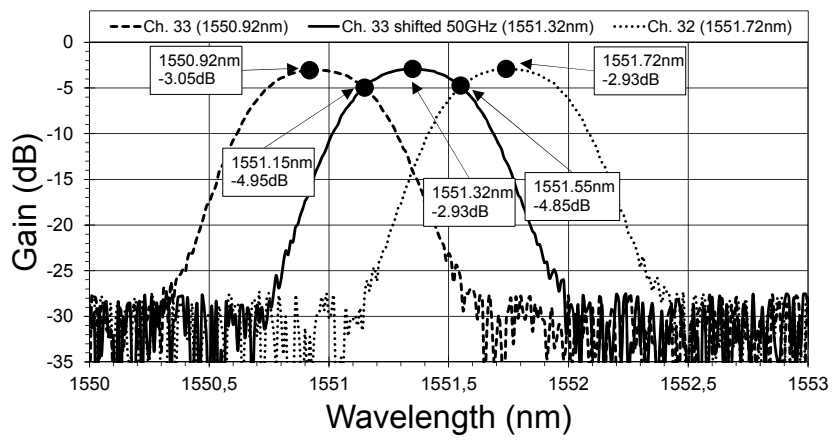

Fig. 9. Interleaving gaussian AWG channels by thermal tuning.

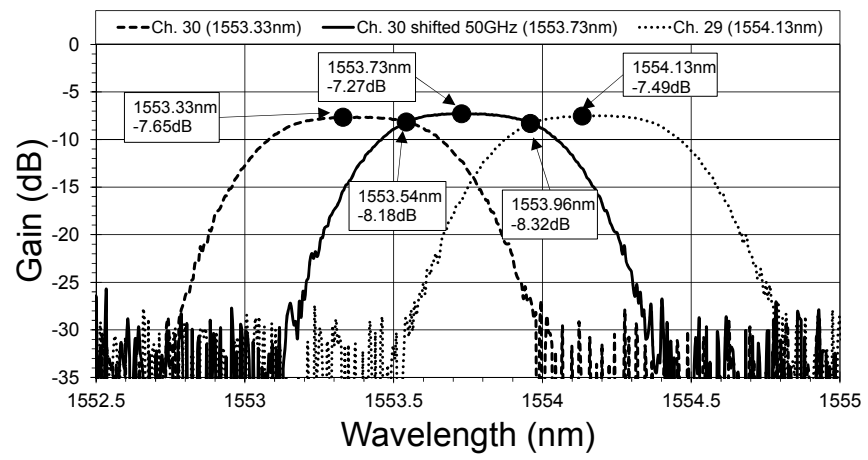

Fig. 10. Interleaving flat-top AWG channels by thermal tuning.

With two flat-top AWGs, by tuning to interleave channels so that one $\mathrm{AWG}$ peaks at the $\mathrm{CP}$ of the other $\mathrm{AWG}$, a ripple of only $0.7 \mathrm{~dB}$ is obtained and a MIL of $8.2 \mathrm{~dB}$ is reached. But using gaussian AWGs, the ripple is $1.9 \mathrm{~dB}$, however the MIL is just $4.9 \mathrm{~dB}$, furnishing the best result.

\section{UDWDM WAVELENGTH ASSIGNMENT}

\section{A. Assignment paradigms and heuristic schemes}

In order to provide a Dedicated WTTU (DWTTU) by spacing channels at few GHZ in the WTTU method, we need to deal with the limited tunability of the lasers. The TLs at the ONU will have random nominal wavelengths in a band with thermal tuning in a window, which is used to assign a wavelength channel without collisions in activation and also during operation processes in a SUDWDM approach. 
We adopt the DWTTU format with a single wavelength for each user by assuming that there is enough optical band to serve all deployed users simultaneously. Another way of exploiting a wavelength channel is the Shared WTTU (SWTTU) pattern, where a wavelength is shared among several users in TDM; the trade-offs between DWTTU and SWTTU will be discussed in section IV.

In the activation process, when an ONU joins or restarts operation in the PON, a previous in-band connection must be established. Several turn-up procedures can be defined, depending on the ONU architectures (one or two lasers) and whether a unique band or two bands are used. Basically, the ONU receiver tunes its TL to a signaling pilot or working wavelength and, after receiving authorization by the OLT, the ONU up-transmits. The OLT, employing MAC messages OLT-ONU, will control by DWA the final channel allocation in activation, and also in operation, when the environmental conditions affect the laser tunable window. An activation protocol was proposed for coherent UDWDM-PON [28], reporting the modifications of the requirements of the XGPON standard, but other protocols are to be developed.

The ONU can hold a TL for downstream and another TL for upstream, both to be assigned, and operating in two different bands or in the same band, or a unique TL for down/up working in a unique band.

Firstly, two assignment paradigms managing the wavelengths in activation and operation are foreseen [29]:

- Static Wavelength Assignment (SWA), where once wavelengths are assigned they remain static.

- Dynamic Wavelength Assignment (DWA), where wavelengths reallocations are made, even to satisfy network changes in environmental temperature conditions.

The ONUs own TLs with wavelengths values randomly distributed in uniform probability in an optical band. The band is divided in equally narrow spaced channels. When an ONU is activated by using SWA, each TL is assigned a free channel in its limited tuning range window, but if there is no channel available in this window, the entrant ONU is rejected. When DWA is used, active working wavelengths can be reassigned to serve a new entering TL that finds all channels busy in its tunable window; in this window a connected user is searched beginning in the lowest channel with at least a free channel in its current window; if this user is found, its working wavelength is reassigned to the free channel and, then, the liberated channel is given to the entering $\mathrm{ONU}$, otherwise the incoming ONU is rejected.

These paradigms are combined with heuristic assignment schemes to practically distribute the ultra-dense spectrum. Three heuristic channel assignment algorithms have been proposed to avoid collisions and efficiently organize the spectrum, suited for the SUDWDM-PON scenario with limited tunability [19]:

- First Fit (FF), allocating the free channel closer to the random ONU wavelength [30].

- Maximum Scattering (MS), selecting the free channel providing maximum distance between two busy channels.

- Maximum Admittance (MA), assigning the free channel giving the maximum options of admission when a future new request arrives at the same assigned channel.

$\mathrm{FF}$ is the most simple assignment scheme, selecting the free channel closer to the original wavelength, and was previously proposed and evaluated for non-preselectedindependent ONU sources with wavelength control [29]. MS algorithm allocates the wavelengths in the band in a more spaced manner, providing free channels more uniformly. Finally, MA scheme keeps free the channels with more probability to be requested in the internal band and at the band limits, where a part of the TL window is out of band and cannot be used; these are the central and side channel band regions, respectively. When using MA scheme, the resulting blocks of assigned channels are smaller than the TL window, and thus, when a novel request arrives over any formed block, the new wavelength can be allocated.

\section{B. Wavelength channel assignment in activation}

The three proposed heuristic assignment algorithms are evaluated by simulations in activation processes, where ONUs join activity in the PON.

The simulations are realized applying the Monte-Carlo method to find the number of channels needed by calculating the ONU rejection probability $P_{r}$, which is the probability of an entrant ONU to be refused because of an inevitable channel collision. The opposite concept is the ONU acceptance probability $P_{a}$, with $P_{a}=1-P_{r}$.

All ONUs present in the PON are assigned randomly one or two wavelengths in a band or two wavelengths in two bands, depending on the deployed scenario, with uniform distribution in the band. The ONUs are tried to be activated sequentially according to the chosen assignment scheme and in a random order. An ONU is rejected when, each ONU owning two TLs, one or both TLs cannot be allocated in their tunable window; or, if there is only one TL per ONU, when this TL cannot be allocated. The number of failures is counted and the process is repeated 10000 times to obtain the average rejection probability.

In a first simulation scenario, each ONU has two TLs in two different bands for down/up, with independent random wavelengths (Fig. 3, a and b). Being $P_{1}$ and $P_{2}$ the rejection probabilities of TL 1 and 2, respectively, with $P_{1}=P_{2} \ll 1$, then the total ONU rejection probability $P_{r}$ is:

$$
P_{r}=P_{1}+P_{2}-P_{1} P_{2} \cong P_{1}+P_{2}=2 P_{1}
$$

Hence, only one band simulation is realized and next the total ONU rejection probability is calculated. When using coherent homodyne detection, the channel spacing is taken to be $6.25 \mathrm{GHz}$ [20], [22], and the required bands can be allocated in the C-band and/or in the L-band. Although the minimum wavelength separation between two adjacent channels is only $3 \mathrm{GHz}$ [20]; considering the guard bands, the wavelengths drifts, the nonlinearities constrains, and future ITU grid, $6.25 \mathrm{GHz}$ are used. The laser tunability is assumed to be $0.1 \mathrm{~nm} /{ }^{\circ} \mathrm{C}$, with a range of $+/-10^{\circ} \mathrm{C}(+/-1 \mathrm{~nm}$ or $+/-20$ channels in $\mathrm{C}$-band), which can be achieved with a Peltier or just by heating the TL by $+10^{\circ} \mathrm{C}$ and, afterwards, incrementing or decrementing its temperature up to $10^{\circ} \mathrm{C}$.

The deployed ONUs are 256, with an ODN split power of 2. FF scheme, being the simplest assignment algorithm, is used as comparison pattern. Simulation results are shown in Fig. 11; with a SWA procedure, MS exceeds FF, but MA 


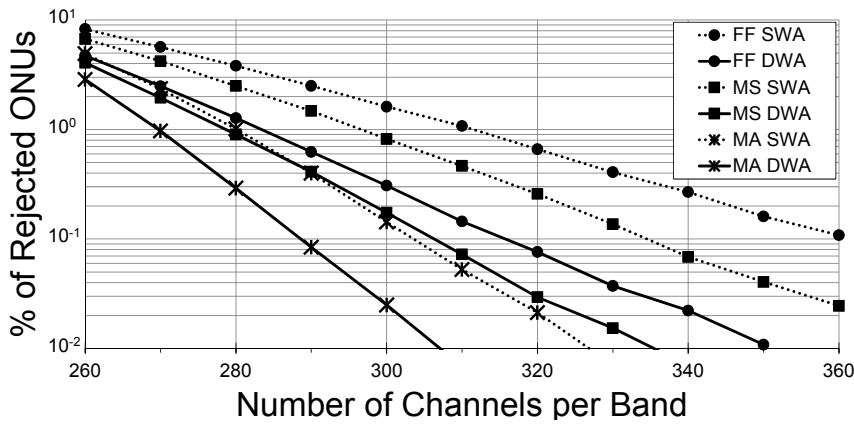

Fig. 11. Two TLs in 2 bands, ONU rejection probability in activation using SWA or DWA paradigms and FF, MS and MA schemes.

provides the best results: an ONU rejection probability of $10^{-3}(0.1 \%$ rejection probability or $99.9 \%$ acceptance probability) is achieved with only 305 channels. With the same ratio, when employing MS, 335 channels are needed; and while using FF, 360 channels per band are required.

Applying the flexible DWA method, where active working channels are reassigned if needed; simulations show a great improvement of the ONU rejection probability (Fig. 11). DWA combined with MA furnishes the best results, with a rejection probability of $0.1 \%$ with only 288 band channels.

Likewise, in a second simulation scenario, both TLs with independent random wavelengths at the ONU share a unique band for down/up (Fig. 3, c and d), with the same $6.25 \mathrm{GHz}$ channelization for coherent detection, 256 active users and identical $+/-10^{\circ} \mathrm{C}$ thermal tuning range. Now, the ONU rejection probabilities exhibit similar outcomes for a required total band slightly greater than the double of channels needed in the previous case with two different bands for up/down. The results for each of the SWA and DWA paradigms and FF, MS and MA schemes are depicted in Fig. 12, being again MA with DWA the best algorithm.

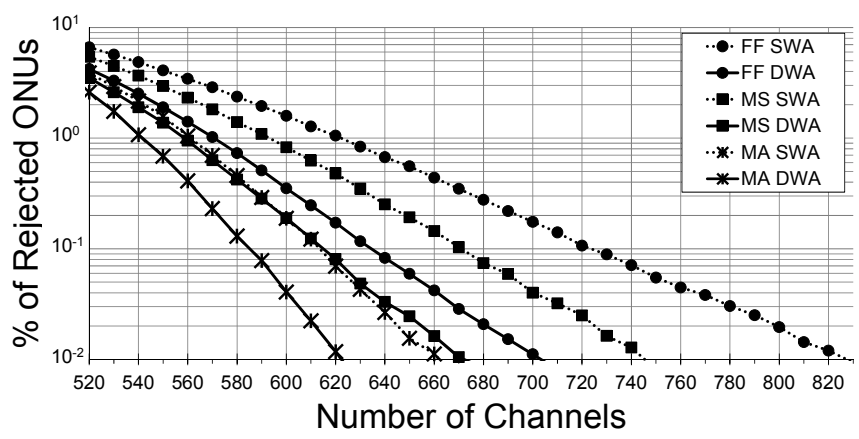

Fig. 12. Two TLs in one unique band, ONU rejection probability in activation using SWA or DWA and FF, MS and MA schemes.

Finally, in a third scenario, there is only one TL for down/up at the ONU in a sole band, as in the previously presented heterodyne detection diagram (Fig. 4), but the channel is doubled to $12.5 \mathrm{GHz}$. The simulation outcomes are equivalent to having only one TL in the band but with a lower tunability of $+/-5^{\circ} \mathrm{C}$, the half of the previous range. The obtained ONU rejection probabilities depict that the total bandwidth needed is greater compared to the former case of having two TLs in the same band, because of using a

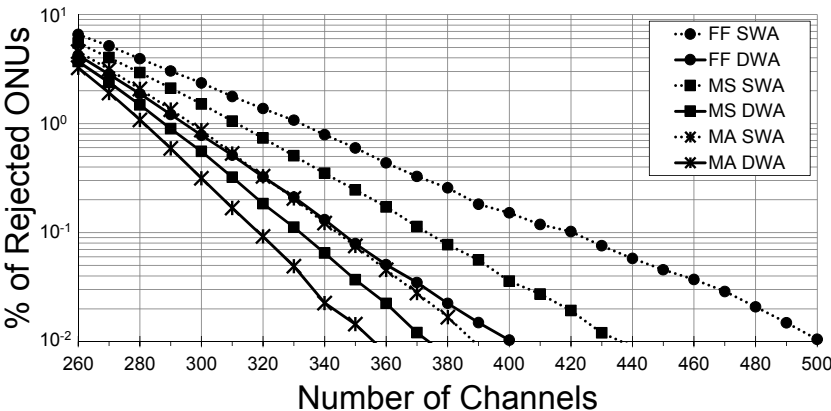

Fig. 13. One TL in one unique band, ONU rejection probability in activation using SWA or DWA and FF, MS and MA schemes.

doubled channelization and now the number of channels are multiplied by $12.5 \mathrm{GHz}$. The results for SWA and DWA paradigms and FF, MS and MA schemes are illustrated in Fig. 13, being once more MA with DWA the best algorithm.

This latest scenario is also equivalent to disposing a coherent homodyne detection employing two paired TLs in the same band for down/up, with different wavelengths and a fixed difference of an odd number of channels.

Simulations are realized for different numbers of users, which are a typical ODN split power of 2 , and for the former scenarios considered. Table I summarizes the required number of channels and bands for the different cases, considering an ONU rejection probability of $0.1 \%$ and using DWA with the best MA scheme. Channels bandwidths are of $6.25 \mathrm{GHz}$ or $12.5 \mathrm{GHz}$ for the homodyne or for the heterodyne and homodyne paired TLs cases, respectively.

TABLE I

REQUIRED ChANNELS IN ACTIVATION PROCEss

\begin{tabular}{|c|c|c|c|}
\hline \multirow[b]{2}{*}{ Users } & \multicolumn{2}{|c|}{ homodyne } & \multirow{2}{*}{$\begin{array}{c}\text { heterodyne \& } \\
\text { homodyne paired TLs } \\
1 \text { TL \& } 2 \text { TLs, } 1 \text { band }\end{array}$} \\
\hline & 2 TLs, 2 bands & $2 \mathrm{TLs}, 1$ band & \\
\hline 32 & $2 \times 32$ & 68 & 34 \\
\hline 64 & $2 \times 68$ & 140 & 75 \\
\hline 128 & $2 \times 141$ & 287 & 156 \\
\hline 256 & $2 \times 288$ & 583 & 317 \\
\hline
\end{tabular}

\section{Operation under environmental conditions}

In an operation scenario, the temperature at the ONU changes under environmental conditions. Commercial operational temperature range for indoor equipment is 5 to $35^{\circ} \mathrm{C}$, but with a moderate variation of few degrees; on the other hand outdoor equipment suffers great temperature changes, as night/day or season shifts exhibit. Hence, when a temperature variation occurs at the ONU, the TL change its tunable window and, when it cannot keep the assigned working channel, a reassignment is needed, which can be realized with a DWA scheme.

The ONU reports the OLT using MAC messages about the tunable window variations; besides, at the OLT an OSA is continuously monitoring all wavelengths to guarantee their correct power and assignment (Fig. 5). Next, when an ONU cannot hold its current assigned channel, the OLT will make decisions about channel reallocations to maintain this ONU under operation. The channel reassignments are realized with any of the former heuristic algorithms and the choices are ordered by MAC messages OLT-ONU. 
To model the temperature variation scenario and show how channel reassignments affect operation, first we deem the case where some ONUs are sited outdoors, subject to great temperature changes, a situation that sometimes occurs in America, placing the ONUs on facades, poles or exterior cabinets. We set the case where $50 \%$ of the ONUs are outdoors and the other $50 \%$ are indoors, suffering great and low deviations, respectively. During a period of 10 days to obtain stable and accurate results, smooth daily changes of $+/-2.5^{\circ} \mathrm{C}$ are applied to indoor ONUs and great variations of $+/-5^{\circ} \mathrm{C}$ to outdoor ONUs, moving the tunable windows of their TLs. Simulations with 256 active ONUs are realized with the same former $+/-10^{\circ} \mathrm{C}$ thermal tunability, owing each ONU two TLs allocated in different bands.

During operation, the TLs which cannot hold their current channel are reassigned, applying any of the DWA schemes. When a TL in an ONU cannot hold its assigned channel, neither be reassigned, the ONU is blocked, waiting for an activation process furnishing a new free channel.

We consider two reallocation cases: first, only one reassignment is produced, changing the present channel to a free channel in the new tunable window (DWA $)$; second, if there is no free channel in the new tunable window, there, a working TL is reallocated if there is a free channel in its current tunable window, liberating a channel which is given to the ONU not holding its current channel $\left(\mathrm{DWA}_{2}\right)$. If an ONU has an assigned channel that is out of tuning and cannot be reassigned, it is out of operation and it is marked as blocked; thus, waiting for a new activation process with channel availability. The percentage of ONUs being not operative at any time, independently of their blocking time, is expressed as an ONU blocking probability $P_{b}$. A flowdiagram of the reallocation process for both $\mathrm{DWA}_{1}$ and DWA $_{2}$ cases is shown in Fig. 14.

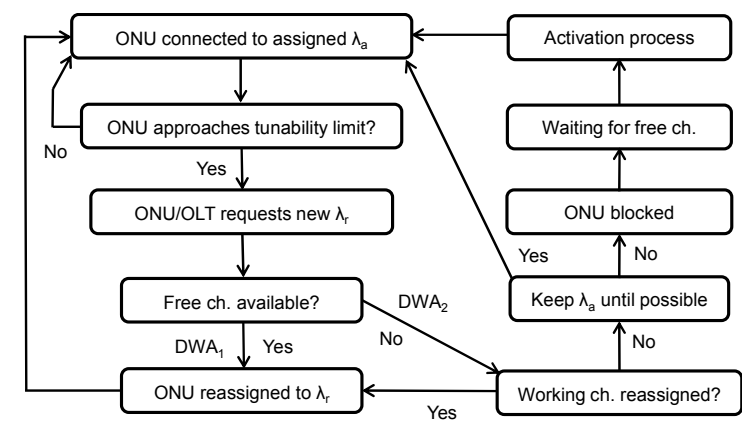

Fig. 14. ONU channel reallocation flow-diagram, by one $\left(\mathrm{DWA}_{1}\right)$ or two $\left(\mathrm{DWA}_{2}\right)$ reassignments, adapting to environmental conditions.

Under these out/indoor temperature variations, an ONU blocking probability of $0.1 \%$ is not reachable, even with 640 channels (32 nm in C-band), as can be seen in Fig. 15. Most of the reassignments or blocking cases are due to the fact that TLs closer to the band limits have a part of their tunable window out of band, and, therefore, they are assigned with low probability and even cannot be assigned.

To avoid a penalty for the TLs with nominal wavelengths near the band limits and improve the ONU blocking probability, we propose to add a guard band, $B_{g}$, at each side of the original band, $B_{a}$, whit the OLT deploying an enlarged reception band, as depicted in Fig. 16.

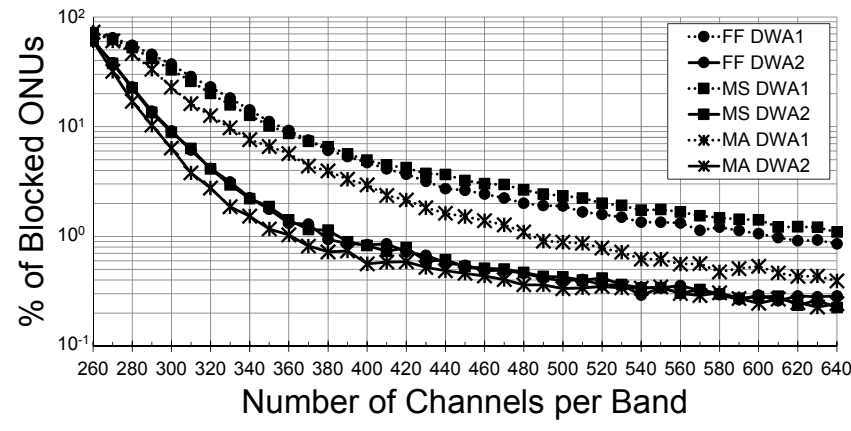

Fig. 15. ONU blocking probability in operation with one $\left(\mathrm{DWA}_{1}\right)$ or two $\left(\mathrm{DWA}_{2}\right)$ reassignments, adapting to environmental conditions.

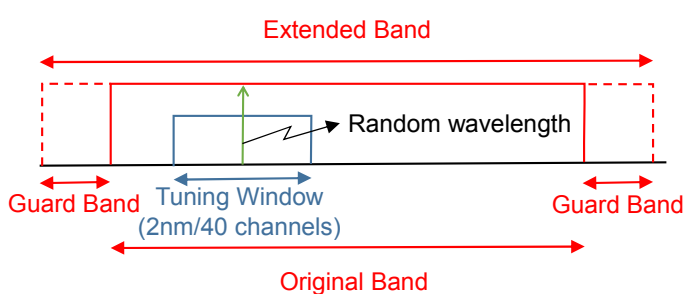

Fig. 16. Guard bands extending the original optical band.

The original band is extended, and the guard bands must be large enough to contain the thermal TL window changes. Hereafter, when adding the guard bands, all TLs always have their tunable windows inside the extended band, $B_{e}$, and, therefore, they have the same opportunities to be accepted. Fig. 17 illustrates the new obtained simulation results with the extended band. The guard bands are of 0.75 $\mathrm{nm}$ (15 channels in C-band) per side for the same out/indoor temperature variations; and, when using MA scheme with $\mathrm{DWA}_{2}$, an ONU blocking probability of $0.1 \%$ is reached with an extended band of only 320 channels.

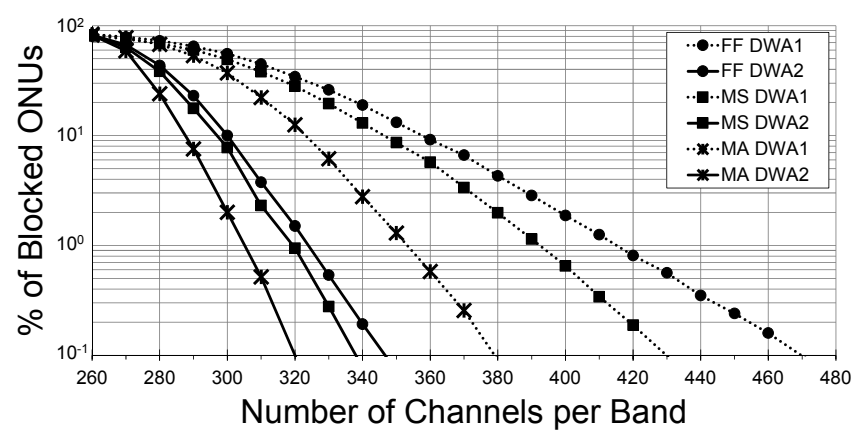

Fig. 17. ONU blocking probability with extended band and with one $\left(\mathrm{DWA}_{1}\right)$ or two $\left(\mathrm{DWA}_{2}\right)$ reassignments, adapting to out/indoor environmental conditions.

Another form to evaluate the scenario under temperature changes is defining the ONU Availability Ratio (OAR), the ratio of ONU available time to total operating time, like as in the transmission systems and media recommendation [31]. Simulating with same former conditions with two reassignments $\left(\mathrm{DWA}_{2}\right), \mathrm{OAR}$ is found for the most blocked ONU and, in Fig. 18, the ONU unavailability ratio (1-OAR), which is the opposite of the OAR, shows that the network adapts dynamically to temperature changes. 


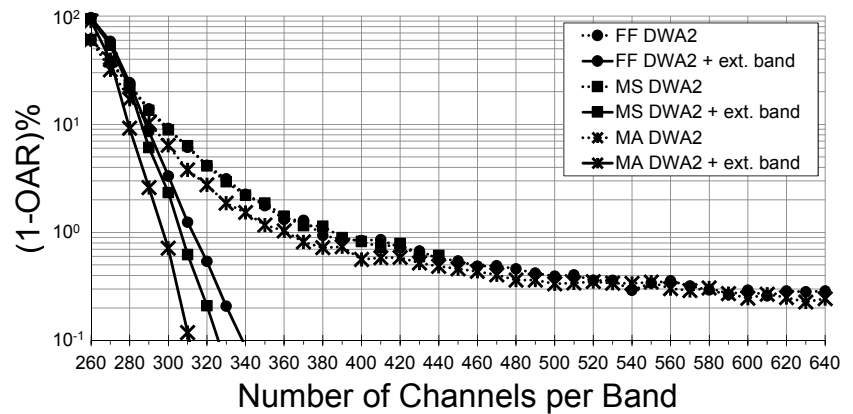

Fig. 18. ONU Availability Ratio (OAR) with and without extended optical band; using $\mathrm{DWA}_{2}$ and FF, MS and MA schemes, adapting to out/indoor environmental temperature changes.

In operation without band extension, the most blocked ONU, which has its TL close to the band limits, suffers an (1-OAR) of $0.8 \%$, even employing more than 500 channels, and independently of the used algorithm. But when the optical band is extended, (1-OAR) drops dramatically, and the MA scheme obtains an OAR of $99.9 \%$ with only 310 channels of extended band, which corresponds to an average time of 86 seconds per day of unavailability for the most blocked ONU.

Simulations with the same daily smooth temperature changes of $+/-2.5^{\circ} \mathrm{C}$ in indoor ONUs and great variations of $+/-5^{\circ} \mathrm{C}$ in outdoor ONUs, and with an equal $+/-10^{\circ} \mathrm{C}$ ONU thermal tunability, are also realized for the former different numbers of users, coherent detection and band scenarios. Table II summarizes the needed number of channels and bands for the different cases considering an OAR of $99.9 \%$ and employing $\mathrm{DWA}_{2}$ with the best MA scheme. Channels bandwidths are of $6.25 \mathrm{GHz}$ or of $12.5 \mathrm{GHz}$ for the homodyne or for the heterodyne and homodyne paired TLs cases, respectively; with an extended optimum band of 8 channels per side.

TABLE II

REQUIRED CHANNELS UNDER OUT/INDOOR OPERATING CONDITIONS

\begin{tabular}{cccc}
\hline \hline & \multicolumn{2}{c}{ homodyne } & $\begin{array}{c}\text { heterodyne \& } \\
\text { homodyne paired TLs }\end{array}$ \\
Users & 2 TLs, 2 bands & 2 TLs, 1 band & 1 TL \& 2 TLs, 1 band \\
\hline 32 & $2 \times 32$ & 77 & 41 \\
64 & $2 \times 77$ & 154 & 84 \\
128 & $2 \times 155$ & 304 & 171 \\
256 & $2 \times 310$ & 623 & 360 \\
\hline \hline
\end{tabular}

A second temperature variation scenario is the case when all ONUs are sited indoors, which is the typical condition in Europe, where FTTH is deployed placing the ONUs at the homes or inside an interior cabinet in a building. Hence, the ONUs suffer low temperature deviations. Smooth dairy changes of $+/-2.5^{\circ} \mathrm{C}$ are applied to indoor ONUs, moving the tunable windows of their TLs. Once more, simulations with 256 active ONUs are realized with the same former $+/-10^{\circ} \mathrm{C}$ ONU thermal tunability, owing each ONU two TLs allocated in different bands.

Fig. 19 shows the new obtained simulation results with the extended band. The guard bands now are taken of 0.25 $\mathrm{nm}$ (5 channels) per side to cover the indoors variations.

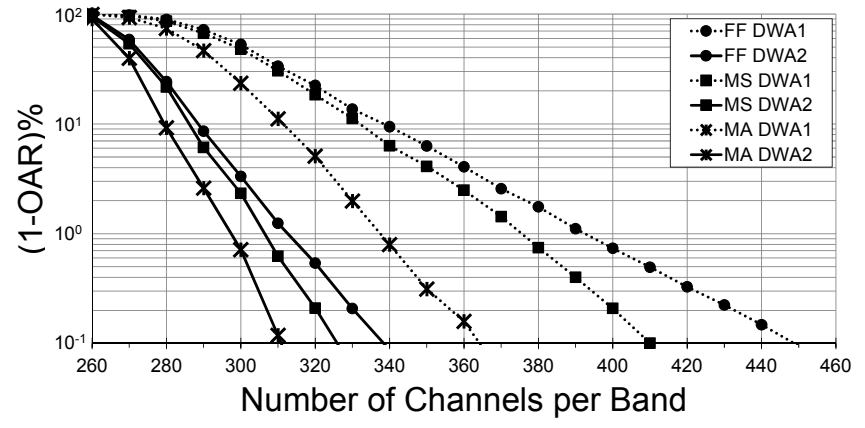

Fig. 19. OAR with extended band and one $\left(\mathrm{DWA}_{1}\right)$ or two $\left(\mathrm{DWA}_{2}\right)$ reassignments, adapting to indoor environmental conditions.

When using MA scheme with $\mathrm{DWA}_{2}$, an ONU blocking probability of $0.1 \%$ is reached with an extended band of 310 channels, equal to the one required for out/indoors.

Simulations with same ONU temperature variations and $+/-10^{\circ} \mathrm{C}$ ONU thermal tunability are executed for the same previous different numbers of users, coherent detection and band scenarios. Table III shows the required number of channels and bands for the different cases, with an OAR of 99.9\% and employing $\mathrm{DWA}_{2}$ with the best MA scheme. In the homodyne cases the channels are of $6.25 \mathrm{GHz}$, with an extended optimal band of 5 channels per side; while for the heterodyne and homodyne paired TLs instances the channels are of $12.5 \mathrm{GHz}$, with an extended optimal band of 3 channels per side. The required channels for indoors are in general lower than the needed for out/indoor conditions.

TABLE III

REQUIRED CHANNELS UNDER INDOOR OPERATING CONDITIONS

\begin{tabular}{|c|c|c|c|}
\hline \multirow[b]{2}{*}{ Users } & \multicolumn{2}{|c|}{ homodyne } & \multirow{2}{*}{$\begin{array}{c}\text { heterodyne } \& \\
\text { homodyne paired TLs } \\
1 \text { TL \& } 2 \text { TLs, } 1 \text { band }\end{array}$} \\
\hline & $2 \mathrm{TLs}, 2$ bands & 2 TLs, 1 band & \\
\hline 32 & $2 \times 32$ & 69 & 37 \\
\hline 64 & $2 \times 69$ & 145 & 84 \\
\hline 128 & $2 \times 145$ & 304 & 166 \\
\hline 256 & $2 \times 310$ & 620 & 356 \\
\hline
\end{tabular}

\section{DIMENSIONING THE NETWORK}

\section{A. Shared versus dedicated wavelength to the user}

When designing the network, two forms of exploiting the wavelength channels must be considered: DWTTU, with a unique wavelength dedicated for each user, and SWTTU, where every wavelength is shared among several users in TDM. The convenience of sharing wavelengths depends on the capacity limits of the PON, which are a function of several factors: the available spectrum to operate, the tuning range of the lasers, the power budget, the receiver sensitivity, the eye power safety and the nonlinearities. If the available optical band is narrow, because of the coexistence with legacy $x P O N$ generations, and the number of deployed ONUs is higher than the offered channels, then SWTTU is mandatory. But if the optical band is wide enough, with more accessible channels than ONUs, DWTTU can be used, and the number of ONUs is only limited by the power budget, eye safety and nonlinearities. 
Therefore, DWTTU is the good choice, because each user can profit the full bit rate in a wavelength (e.g. 1G), transmitting in a continuous stream without burst mode and besides with low-cost transceivers.

The number of needed wavelength channels in the optical bandwidth, $N_{c}$, versus the allocated ONUs, $N_{O N U}$, is evaluated to have a comparison between DWTTU and SWTTU. With a loss budget of $48 \mathrm{~dB}$ and a reach of $45 \mathrm{~km}$, up to 256 ONUs can be served, as it will be shown in next chapter of this section. Considering a $6.25 \mathrm{GHz}$ channel spacing and working in C-band, when two TLs are used in different up/down bands at the ONUs, $N c$ versus $N_{O N U}$ is illustrated in Fig. 20, as a function of the ONU tunability, depending on the activation process and the environmental operation conditions, and deploying up to 256 users.

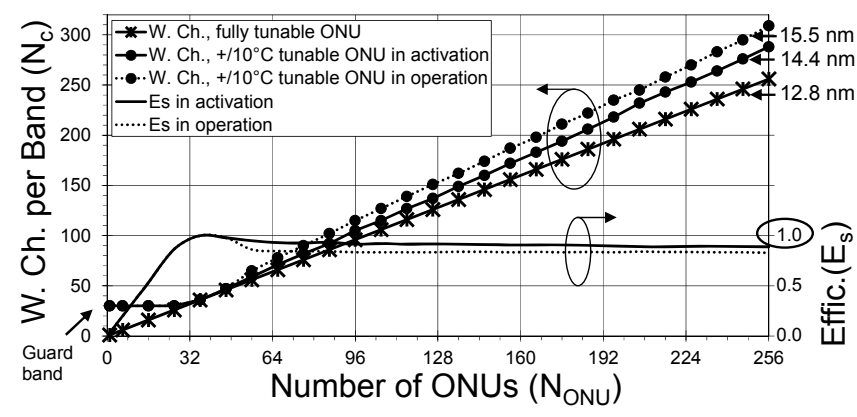

Fig. 20. DWTTU: needed wavelength channels and spectral channel efficiency vs. number of allocated ONUs.

Using DWTTU, with full laser tunability in the bands at the ONUs, $N_{c}$ corresponds to $N_{O N U}$ linearly; therefore, to serve up to 256 users, just 256 channels $(12.8 \mathrm{~nm})$ are needed. However, by employing ONU TLs with a limited tunability of $0.1 \mathrm{~nm} /{ }^{\circ} \mathrm{C}$, a variation of $+/-10^{\circ} \mathrm{C}$ provides a tuning range of $2 \mathrm{~nm}$ or 40 channels; hereafter, in the activation processes, the efficient DWA MA algorithm provides the required number of activation channels, $N_{a}$ (Table I), or the corresponding necessary activation bandwidth, $B_{a}$, which is increased, and 288 channels (14.4 nm) are needed to cover all 256 users. With the same assignment scheme in out\&indoor operating conditions, the required number of operation channels, $N_{o}$, or the equivalent extended operation bandwidth, $B_{o}$, are now augmented up to 310 channels or $15.5 \mathrm{~nm}$, respectively (Tables II and III).

The spectral channel efficiency, defined as the number of ONUs over the needed channels, $E_{s}=N_{O N U} / N_{c}$, is in the order of $90 \%$ in activation and about $80 \%$ in operation (Fig. $20)$. In the SWTTU case, we may have $E_{s}>1$ with 256 active users, but employing less working channels than users.

The sharing factor, $F_{s}$, which is the number of ONUs working in the same channel in TDM, and the served bit rate per user in a channel relative to $1 \mathrm{G}, R_{u}$, are shown in Fig. 21. As the number of shared wavelengths decreases, the greater is $F_{s}$, but the lower is the available $R_{u}$ for each ONU. When no wavelengths are shared between users, we have DWTTU; hence, $F_{s}$ and $R_{u}$ take the unit value.

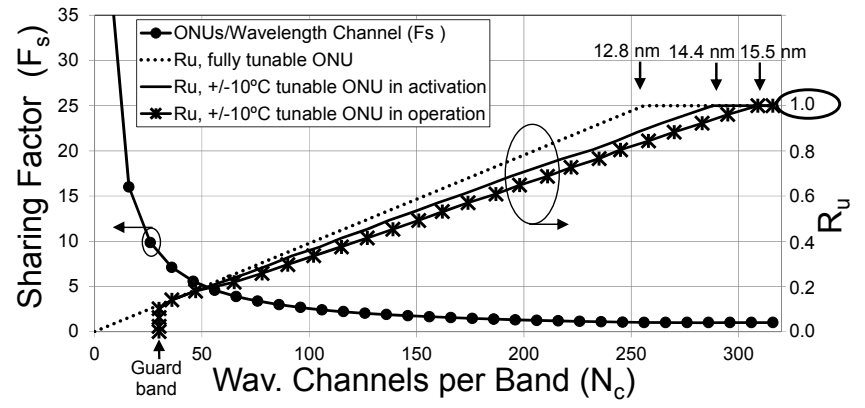

Fig. 21. SWTTU: sharing factor and bit rate per user in a channel relative to $1 \mathrm{G}$ vs. employed wavelength channels.

\section{B. Spreading DFB-lasers over the optical band}

Low-cost DFB-lasers suffer manufacturing wavelength variations, which are mainly due to microscopic cleaving deviations. The yielded central nominal wavelength is dispersed approximately $+/-1 \mathrm{~nm}$ in a scattering bandwidth, $B_{s}$, of about $2 \mathrm{~nm}$, exhibiting an estimated exponential distribution [15]. In order to provision a set of TLs with wavelengths uniformly distributed in a whole optical band, DFB-lasers with scattered wavelengths are produced in subbands with a $B_{s} / 2$ spacing and, gathering them by overlapping the sub-bands, the arranged set of lasers provides wavelengths with an approximated uniform distribution in a complete band (Fig. 2). The number of needed sub-bands, $L_{a}$, to generate an approached uniform distribution in the activation band, $B_{a}$, is:

$$
L_{a}=\left\lfloor 2 B_{a} / B s\right\rfloor
$$

In equation (2), $\lfloor x\rfloor$ is the floor function, delivering the largest integer not greater than $x$. To furnish TLs to 256 deployed ONUs, with wavelengths uniformly distributed in the band, when two TLs are used in different bands, the required activation band is of $N_{a}=288$ channels (Table I) (or $B_{a}=$ $14.4 \mathrm{~nm}$ ). Thus $L_{a}=14$ sub-bands with $B_{s}=2 \mathrm{~nm}$ are enough for each up and down bands to provide the ONUs.

The required number of $2 \mathrm{~nm}$ width activation sub-bands in C-band to have a set a of TLs to provide the ONUs with wavelengths uniformly distributed in the activation band for the different architecture cases are summarized in Table IV, with $B_{a}$ providing a $0.1 \%$ ONU rejection probability by using DWA with the MA scheme, as shown in Table I.

TABLE IV

REQUIRED NUMBER OF ONU ACTIVATION SUB-BANDS

\begin{tabular}{|c|c|c|c|}
\hline \multirow[b]{2}{*}{ Users } & \multicolumn{2}{|c|}{ homodyne } & \multirow{2}{*}{$\begin{array}{l}\text { heterodyne \& } \\
\text { homodyne paired TLs } \\
1 \text { TL \& } 2 \text { TLs, } 1 \text { band }\end{array}$} \\
\hline & 2 TLs, 2 bands & 2 TLs, 1 band & \\
\hline 32 & $2 \times 1$ & 3 & 3 \\
\hline 64 & $2 \times 3$ & 7 & 7 \\
\hline 128 & $2 \times 7$ & 14 & 15 \\
\hline 256 & $2 \times 14$ & 29 & 31 \\
\hline
\end{tabular}

When implementing the OLT for DWTTU with ONUs under limited tunability, the number of required operation channels, $N_{o}$, is defined by the environmental conditions. With two TLs at the ONU in different up/down bands and under out/indoor circumstances, to provide an 99.9\% OAR, 
$N_{o}=310$ channels are needed to serve 256 users allocated using the $\mathrm{DWA}_{2}$ MA scheme (Table III), with an extended operation band, $B_{o}$, corresponding to $15.5 \mathrm{~nm}$ (Fig. 21).

One option is to use at the OLT a number of TLs, $N_{t}$, with full tunability in the operation band. For a number of working ONUs, $N_{O N U}$, a total quantity of $N_{t}=N_{O N U}$ TLs with full tunability in the operation band are necessary. Thus, 256 TLs being full tunable at $B_{o}=15.5 \mathrm{~nm}$ cover all 256 deployed ONUs, but this is a very expensive solution.

Hereafter, the same manufactured DFB-lasers to serve the deployed ONUs in sub-bands can be used for a low-cost OLT implementation just adding new sub-bands, and also furnishing a guaranteed non-blocking structure at the OLT.

Note that outdoors ONUs can suffer harsh temperature changes and their TLs move widely the tuning range, while at the OLT the TLs remain under ambient temperature of about $25^{\circ} \mathrm{C}$. The required number of operation sub-bands is:

$$
L_{o}=\left\lceil 2 B_{o} / B_{t}\right\rceil
$$

In equation (3), $\lceil x\rceil$ is the ceiling function, delivering the smallest integer not less than $x$, and $B_{t}$ is the DFB-laser tuning range, which is of $2 \mathrm{~nm}$ or 40 channels in C-band for a variation of $+/-10^{\circ} \mathrm{C}$ with a $0.1 \mathrm{~nm} /{ }^{\circ} \mathrm{C}$ tunability, being equal to the scattering range $B_{s}$. Hence, to cope with ONUs owning two TLs in different up/down bands and under out/indoor circumstances, an operation band of $15.5 \mathrm{~nm}$ requires a distribution of DFB-lasers in $L_{o}=16$ sub-bands.

Table V shows the required number of OLT operation sub-bands under out/indoor circumstances for the different architecture instances that we are considering, with an ONU blocking probability of $0.1 \%$ and employing $\mathrm{DWA}_{2}$ with the best MA scheme (Table II):

Hence, the number of needed lasers per sub-band will be:

$$
N_{s}=\left\lceil N_{o} / L_{o}\right\rceil
$$

With $N_{o}$ channels of $6.25 \mathrm{GHz}$. We require $N_{s}=\lceil 310 / 16\rceil=$ 20 TLs per sub-band. Then, the total OLT TLs per band are:

$$
N_{l}=L_{o} N_{s}
$$

Therefore, we implement $N_{l}=16 \times 20=320 \mathrm{TLs}$ per each up and down band at the OLT. Table VI summarizes the required number of TLs at the OLT for the different architecture instances that we are taking into account.

Considering the indoors most current environment conditions, where all ONUs are located inside homes, the results found for the number of OLT operation sub-bands (Table VII) and the needed TLs at the OLT (Table VIII) are very similar because of the rounding of the ceiling function

\begin{tabular}{|c|c|c|c|}
\hline \multirow[b]{2}{*}{ Users } & \multicolumn{2}{|c|}{ homodyne } & $\begin{array}{c}\text { heterodyne \& } \\
\text { homodyne paired TLs }\end{array}$ \\
\hline & 2 TLs, 2 bands & 2 TLs, 1 band & 1 TL \& 2 TLs, 1 band \\
\hline 32 & $2 \times 2$ & 4 & 5 \\
\hline 64 & $2 \times 4$ & 8 & 9 \\
\hline 128 & $2 \times 8$ & 16 & 18 \\
\hline 256 & $2 \times 16$ & 32 & 36 \\
\hline
\end{tabular}

TABLE V

REQUIRED NUMBER OF OLT OUT/INDOOR OPERATION SUB-BANDS
TABLE VI

\begin{tabular}{|c|c|c|c|}
\hline \multirow[b]{2}{*}{ Users } & \multicolumn{2}{|c|}{ homodyne } & \multirow{2}{*}{$\begin{array}{c}\text { heterodyne } \& \\
\text { homodyne paired TLs } \\
1 \text { TL \& } 2 \text { TLs, } 1 \text { band }\end{array}$} \\
\hline & $2 \mathrm{TLs}, 2$ bands & $2 \mathrm{TLs}, 1$ band & \\
\hline 32 & $2 \times 32$ & 80 & 85 \\
\hline 64 & $2 \times 80$ & 160 & 171 \\
\hline 128 & $2 \times 160$ & 304 & 342 \\
\hline 256 & $2 \times 320$ & 640 & 720 \\
\hline
\end{tabular}

REQUIRED NUMBER OF OUT/INDOOR OLT OPERATION TUNABLE LASERS UNDER LIMITED TUNABILITY

\begin{tabular}{|c|c|c|c|}
\hline \multirow[b]{2}{*}{ Users } & \multicolumn{2}{|c|}{ homodyne } & \multirow{2}{*}{$\begin{array}{c}\text { heterodyne } \& \\
\text { homodyne paired TLs } \\
1 \text { TL \& } 2 \text { TLs, } 1 \text { band }\end{array}$} \\
\hline & 2 TLs, 2 bands & $2 \mathrm{TLs}, 1$ band & \\
\hline 32 & $2 \times 2$ & 4 & 5 \\
\hline 64 & $2 \times 4$ & 8 & 9 \\
\hline 128 & $2 \times 8$ & 16 & 17 \\
\hline 256 & $2 \times 16$ & 31 & 36 \\
\hline
\end{tabular}

TABLE VII

REQUIRED NUMBER OF OLT INDOOR OPERATION SUB-BANDS

TABLE VIII

REQUIRED NUMBER OF OLT INDOOR OPERATION TUNABLE LASERS UNDER LIMITED TUNABILITY

\begin{tabular}{cccc}
\hline & \multicolumn{2}{c}{ homodyne } & $\begin{array}{c}\text { heterodyne \& } \\
\text { homodyne paired TLs }\end{array}$ \\
Users & 2 TLs, 2 bands & 2 TLs, 1 band & 1 TL \& 2 TLs, 1 band \\
\hline 32 & $2 \times 32$ & 72 & 75 \\
64 & $2 \times 72$ & 152 & 171 \\
128 & $2 \times 152$ & 304 & 340 \\
256 & $2 \times 320$ & 620 & 720 \\
\hline \hline
\end{tabular}

\section{Sizing the OLT architectures}

Two OLT architectures have been proposed in Section II chapter B: a) with splitters and a circulator and b) with thermal AWGs combined with splitters at the AWG ports.

In the case a) the OLT is designed filterless with splitters (Fig. 5, a). Using $N_{t}$ full-tunable TLs in the operation band, the implementation has a total split, $S_{t}$, mirroring the deployed split at the ODN (Fig. 1), with a splitting ratio $S_{t}=$ $N_{t}=N_{O N U}$ at the dedicated branches for up and down, which are separated by a circulator. For example, with $N_{O N U}=$ 256 , a two stage split of $16 \times 16$ at each branch can be deployed; and with $N_{O N U}=128,64$ or 32 , a two stage split at each branch can be of $8 \times 16,4 \times 16$ or $2 \times 16$, respectively.

But when we deploy TLs distributed in operation subbands of limited tunability, the number of needed TLs is generally greater than the number on ONUs, $N_{l}>N_{O N U}$; and, thus, at the OLT a next higher level of split, $S_{l}$, compared to the ODN split is needed. Then, usually $S_{l}>N_{l}$ $>$ NONU. For 256 ONUs, in the out/indoor case with two TLs in different up/down bands, the number of TLs per band at the OLT is $N_{l}=320 \mathrm{TLs}$, and four of the 16-split at each branch can have another 2 -split and the TLs able to connect will be $256+4 \times 16=320$. Or simply a three stage split of $S_{l}$ $=2 \times 16 \times 16=512$ can be built. Similar results are obtained for 128, 64 or 32 deployed ONUs and dealt cases (Table IX).

Likewise, for the indoor environmental scenario, identical results as in Table IX are obtained and same split ratios at the OLT using TLs under limited tunability are needed. 
TABLE IX

REQUIRED OLT SPLIT RATIO FOR OUT/INDOOR AND INDOOR OPERATION WITH LASERS UNDER LIMITED TUNABILITY

\begin{tabular}{cccc}
\hline \hline & \multicolumn{2}{c}{ homodyne } & $\begin{array}{c}\text { heterodyne \& } \\
\text { homodyne paired TLs }\end{array}$ \\
Users & 2 TLs, 2 bands & 2 TLs, 1 band & 1 TL \& 2 TLs, 1 band \\
\hline 32 & $2 \times 32$ & 128 & 128 \\
64 & $2 \times 128$ & 256 & 256 \\
128 & $2 \times 256$ & 512 & 512 \\
256 & $2 \times 512$ & 1024 & 1024 \\
\hline \hline
\end{tabular}

The number of lasers with limited tunability to be implemented at the splitters of the OLT is indicated in Tables VI and VIII, and distributed in the corresponding sub-bands as specified in Tables V and VII, depending on out/indoors or indoors deployments, respectively.

In the other case b) the OLT architecture combines two thermal AWGs with splitters at the AWG ports (Fig. 5, b). The AWG channel port spacing is taken of $W \mathrm{GHz}$, being $M$ the available ports at the AWG. The number of AWG required ports per band is:

$$
M_{r}=\left\lceil B_{o} / W\right\rceil
$$

For 256 users with two TLs in different up/down bands in the out/indoor case, a $100 \mathrm{GHz}$ AWG port spacing and an operation band of 310 channels, we need $\lceil 310 \times 6.25 \mathrm{GHz} / 100$ $\mathrm{GHz}\rceil=20$ ports. Then, employing a 41 -port AWG, 20 ports can be devoted for down and other 20 ports for up.

If we try to use full-tunable lasers in the band at the OLT, because of the AWG, the lasers connected to one port just can tune the own port bandwidth, therefore a tunability of only $2 \mathrm{~W} \mathrm{GHz}$ in each port is required, because of the thermal AWG channel interleaving. Hence, TLs with limited tunability, such as DFB-lasers, can be employed, providing a tuning range $B_{t}$ of $2 \mathrm{~nm}$ or $250 \mathrm{GHz}$ in C-band for a $0.1 \mathrm{~nm} /{ }^{\circ} \mathrm{C}$ tunability and with a $+/-10^{\circ} \mathrm{C}$ temperature variation. Then, with $B_{t}>2 \mathrm{~W}$, low-cost DFB TLs scattered in operation sub-bands can be used instead of full-tunable lasers in the whole band. Hereafter, considering a number of $N_{o}$ TLs, which are specified in Table VI, the number of lasers per AWG port implementing two AWGs, is:

$$
N_{p}=\left\lceil N_{o} / 2 M_{r}\right\rceil
$$

Then, the number of TLs per band at each AWG is:

$$
N_{b}=M_{r} N_{p}
$$

Hence, the split ratio, $S_{p}$, deployed at each port will be the number power of 2 greater than $N_{p}$. With 310 operation channels, we need $N_{p}=\lceil 310 /(2 \times 20)\rceil=8 \mathrm{TLs} /$ port, and therefore an 8-split at each port of both AWGs is enough. Then, the number of TLs per band is $N_{b}=20 \times 8=160$, and for two bands a total number of 320 lasers is needed. The required laser tunability is of $2 \mathrm{~W}=200 \mathrm{GHz}$ for lasers with wavelengths centered at each AWG-port frequency.

The required number of operation sub-bands $L_{o}$ to distribute the TLs is stated in equation (3), and specified in Tables V and VII, depending on out/indoors or indoors deployments, respectively. The number of required lasers per band $N_{b}$ is expressed in equation (8). The TLs are distributed along the splitters connected to the ports of both thermal AWGs, assigning to each port the sub-band nearest to the central wavelength of the port.

In summary, for the considered 256 users and under an out/indoor environment with an operation band of $N_{o}=310$ channels, we need 160 TLs per band in an AWG or a total of 320 TLs per AWG, so a total of 640 TLs at the OLT.

We implement two $100 \mathrm{GHz}$ 41-port thermal AWGs with 20 ports devoted for up and 20 ports dedicated for down and with 8 TLs per port, being an 8-split at each port sufficient. We provide 16 optical sub-bands with 20 DFB TLs per subband to cover the total of 320 TLs for up and other 16 subbands with 20 DFB TLs per sub-band for down, which are distributed along both AWG-ports.

The remaining ports at both AWGs can be used to control thermally their tuning. A pilot reference laser with a known stable wavelength is injected to the 50/50 coupler reaching both AWG ports (Fig. 5, b); then, the AWG tunings are moved in such a way that when a receiver in one AWG port detects a maximum pilot power, at the port of the other AWG a minimum pilot signal is received. This assures that both AWGs have complementary frequency responses.

Similar results for the number of ports and lasers can be achieved for 128, 64 or 32 deployed ONUs, with different 50 or $25 \mathrm{GHz}$ AWG channel port spacing and for all considered transmission cases. Table X and XI illustrate the number of ports per band for each AWG and the number of TLs per port for out/indoor and outdoor conditions, respectively.

TABLE X

\begin{tabular}{|c|c|c|c|c|c|c|c|}
\hline \multirow[t]{2}{*}{ Users } & \multirow[t]{2}{*}{$\begin{array}{c}\text { AWG } \\
\text { port } \\
\text { Spac } \\
\text {-ing } \\
\mathrm{GHz}\end{array}$} & \multicolumn{4}{|c|}{ homodyne } & \multicolumn{2}{|c|}{$\begin{array}{l}\text { heterodyne } \\
\& \text { homodyne } \\
\text { paired TLs } \\
1 \text { TL \& } \\
2 \text { TLs } \\
1 \text { band }\end{array}$} \\
\hline & & ports & $\mathrm{TLs} / \mathrm{p}$ & ports & $\mathrm{TLs} / \mathrm{p}$ & ports & $\mathrm{TLs} / \mathrm{p}$ \\
\hline 32 & 25 & $2 \times 8$ & 2 & 20 & 2 & 21 & 2 \\
\hline 32 & 50 & $2 \times 4$ & 4 & 10 & 4 & 11 & 4 \\
\hline 64 & 25 & $2 \times 20$ & 2 & 39 & 2 & 42 & 2 \\
\hline 64 & 50 & $2 \times 10$ & 4 & 20 & 4 & 21 & 4 \\
\hline 128 & 50 & $2 \times 20$ & 4 & 38 & 4 & 43 & 4 \\
\hline 128 & 100 & $2 \times 10$ & 8 & 19 & 8 & 22 & 8 \\
\hline 256 & 100 & $2 \times 20$ & 8 & 39 & 8 & 45 & 8 \\
\hline
\end{tabular}

REQUIRED OLT AWG PORTS AND TLS PER PORT FOR OUT/INDOOR OPERATION WITH LASERS UNDER LIMITED TUNABILITY

TABLE XI

\begin{tabular}{|c|c|c|c|c|c|c|c|}
\hline \multirow[t]{2}{*}{ Users } & \multirow[t]{2}{*}{$\begin{array}{c}\text { AWG } \\
\text { port } \\
\text { Spac } \\
\text {-ing } \\
\text { GHz }\end{array}$} & \multicolumn{4}{|c|}{ homodyne } & \multicolumn{2}{|c|}{$\begin{array}{l}\text { heterodyne } \\
\& \text { homodyne } \\
\text { paired TLs } \\
1 \text { TL \& } \\
2 \text { TLs } \\
1 \text { band }\end{array}$} \\
\hline & & ports & TLs/p & ports & $\mathrm{TLs} / \mathrm{p}$ & ports & $\mathrm{TLs} / \mathrm{p}$ \\
\hline 32 & 25 & $2 \times 8$ & 2 & 18 & 2 & 19 & 2 \\
\hline 32 & 50 & $2 \times 4$ & 4 & 9 & 4 & 10 & 4 \\
\hline 64 & 25 & $2 \times 18$ & 2 & 37 & 2 & 42 & 2 \\
\hline 64 & 50 & $2 \times 9$ & 4 & 19 & 4 & 21 & 4 \\
\hline 128 & 50 & $2 \times 19$ & 4 & 38 & 4 & 42 & 4 \\
\hline 128 & 100 & $2 \times 10$ & 8 & 19 & 8 & 21 & 8 \\
\hline 256 & 100 & $2 \times 20$ & 8 & 39 & 8 & 45 & 8 \\
\hline
\end{tabular}

REQUIRED OLT AWG PORTS AND TLS PER PORT FOR INDOOR OPERATION WITH LASERS UNDER LIMITED TUNABILITY 

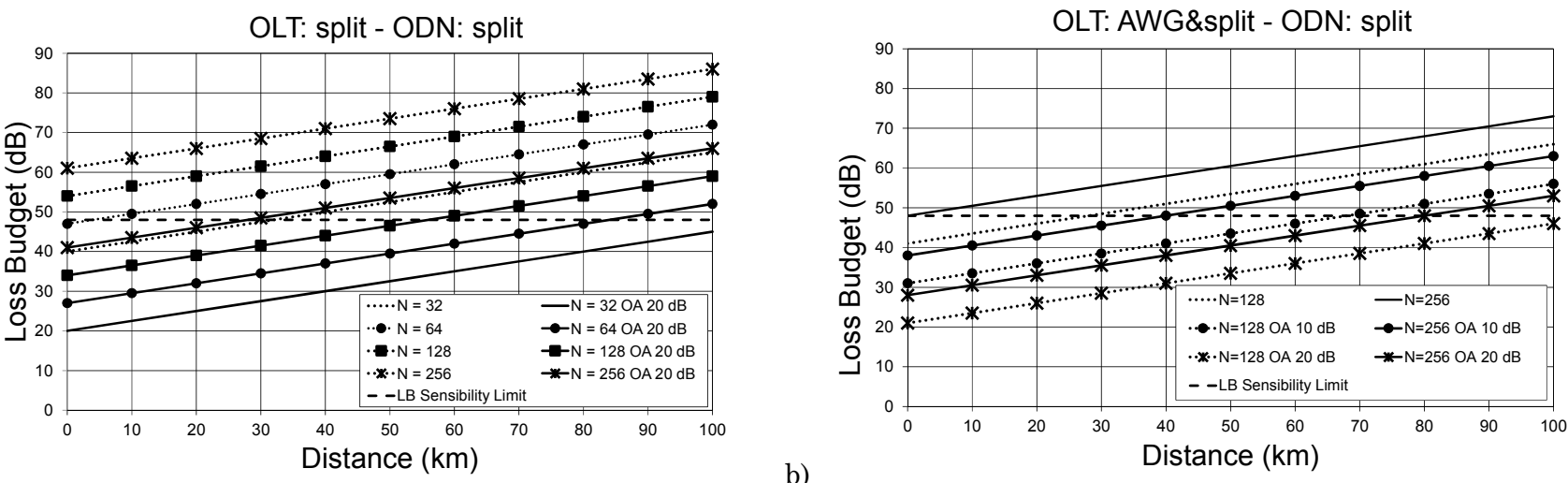

a)

Fig. 22. a) Loss Budget and distance reach for OLT architectures with DFB-lasers under limited tunability distributed in sub-bands: a) splitters and a circulator, b) two thermal AWGs combined with splitters.

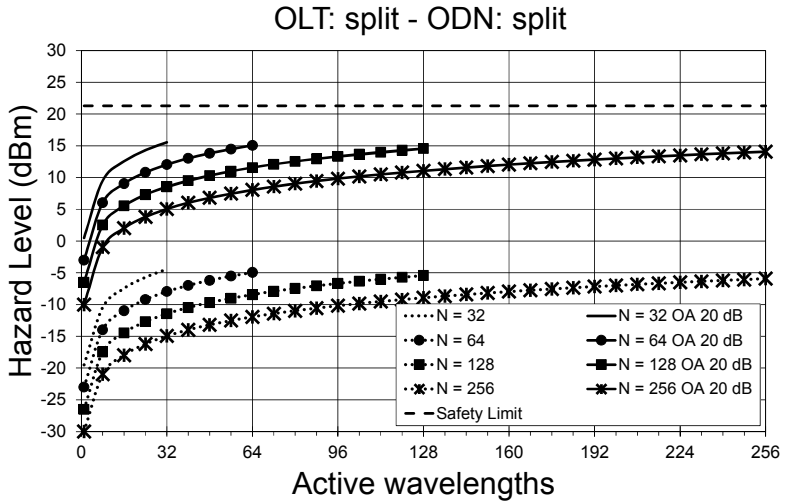

a)

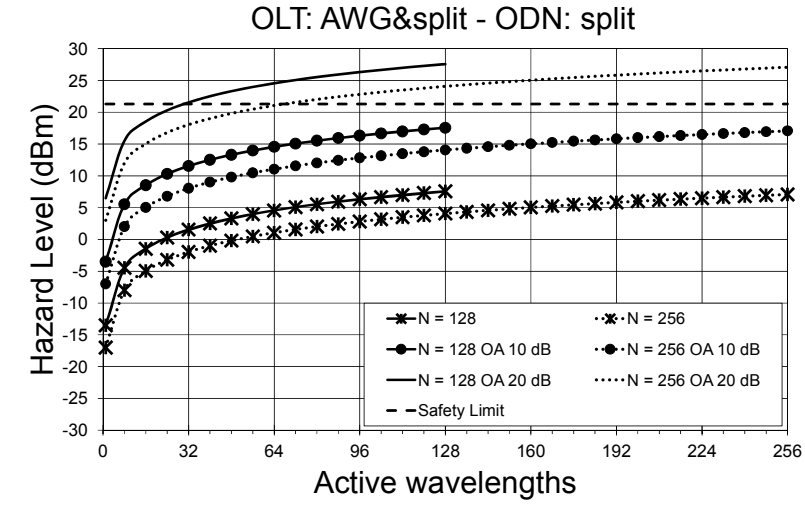

b)

Figure 23. a) Launched power versus number of active wavelengths and eye safety limit for OLT architectures with DFB-lasers under limited tunability distributed in sub-bands: a) splitters and a circulator, b) two thermal AWGs combined with splitters.

\section{System reach, power safety and nonlinearities}

In order to evaluate the network characteristics and its limits we will take into account the complete network and all the involved devices. The ODN is built with optical fibers and splitters (Fig. 1) and we consider again both proposed OLT architecture cases, as depicted in Fig. 5: a) with splitters and a circulator, and b) with two thermal AWGs combined with splitters at the AWG ports.

The first calculated parameter is the system reach, depending on the Loss Budget (LB), which is the amount of signal attenuation that the system will suffer between the transmitter and the receiver, where we will deem not only the ODN but also the internal OLT architecture.

The estimated receiver sensitivity of a coherent reception is of $-45 \mathrm{dBm}$ at both corresponding sides ONU/OLT for the proposed ONU architectures cases [18], [20], [25] and we suppose that ONU/OLT transmitters launch a power of 3 $\mathrm{dBm}$; therefore, we have a maximum $\mathrm{LB}$ of $48 \mathrm{~dB}$ at both ONU/OLT sides at the sensitivity limit.

The optical fiber attenuation in the ODN is taken to be of $0.25 \mathrm{~dB} / \mathrm{km}$, and each 2 -split in the ODN (and also inside the OLT) has a total IL of $3.5 \mathrm{~dB}$, because an Excess Loss (EL) of $0.5 \mathrm{~dB}$ is considered. The asymmetric coupler (90/10), which is inserted in the PON trunk giving access to the OSA, introduces an IL of $1 \mathrm{~dB}$.
In the OLT splitter based architecture case a), by using DFB TLs, a next higher level of split compared to the ODN split is implemented. The circulator, separating up from down transmission branches, has an IL of $0.5 \mathrm{~dB}$.

In the architecture instance b) disposing two thermal AWGs combined with splitters at the AWG ports, the AWGs are chosen of gaussian type, because they furnish the lowest MIL at the interleaved CP of the spectral response, taking an AWG IL of $5.0 \mathrm{~dB}$ in the calculations. For 256 users, the AWG channel port spacing is of $100 \mathrm{GHz}$ with an 8-split per port; while for 128 users is taken of $50 \mathrm{GHz}$ with a 4 -split per port, therefore furnishing a better LB. The lowest LB is achieved with a port spacing of only $25 \mathrm{GHz}$ with a 2 -split level per port, which can be used for 64 or 32 users.

The calculated LBs for both OLT architecture designs, with and without $\mathrm{OA}$ of 10 or $20 \mathrm{~dB}$, are illustrated in Fig. 22. Hence, in split based OLT (case a), with a 32 -split ODN only $30 \mathrm{~km}$ are reached without OA, but with $20 \mathrm{~dB}$ OA a 256 -split attains $30 \mathrm{~km}$ and a 128-split reaches up to $55 \mathrm{~km}$.

Using AWGs combined with splitters at the AWG-ports (case b) improves dramatically the LB. Now, without OA and with a 128-split ODN up to $30 \mathrm{~km}$ are attained, and with $10 \mathrm{~dB}$ OA and same 128-split, $70 \mathrm{~km}$ are reached. For a 256-split ODN, we need $10 \mathrm{~dB}$ OA to accomplish $40 \mathrm{~km}$ and $20 \mathrm{~dB}$ OA to reach $80 \mathrm{~km}$. If only 64 users are deployed, 55 $\mathrm{km}$ are achieved without $\mathrm{OA}$ and 95 with $10 \mathrm{~dB}$ OA. 
The Hazard Level (HL) or eye power safety, which is the maximum allowed power to avoid a risk exposure, is to be considered in any type of locations: unrestricted (domestic and public areas), restricted (not open to the public such as $\mathrm{CO}$ ) and controlled (cable ducts, street cabinets).

The maximum HL allowed or safety limit will be of class $1 \mathrm{M}$, with level of radiation safe under normal operating conditions; for Single Mode (SM) operation in $1550 \mathrm{~nm}$ band, this level is $136 \mathrm{~mW}(21.34 \mathrm{dBm})$ [32]. HL is to be considered as the number of working channels increases and the most critical point is the trunk of the ODN feeder, which is a restricted location in the $\mathrm{CO}$ or a controlled site in a street cabinet.

The launched power to the ODN trunk as a function of active wavelengths, together with the safety limit, is represented in Fig. 23 for both proposed OLT architectures.

In the OLT splitter based architecture case a), the HL is always below the safety limit, even employing a $20 \mathrm{~dB}$ OA. By deploying at the OLT two thermal AWGs combined with splitters case b), the power is also below the safety limit if the OA has a maximum gain of $10 \mathrm{dBs}$.

Finally, the nonlinearities, which do not depend on the number of active wavelengths for a number greater than 64 , limit the feeder power per channel below $-2 \mathrm{dBm}$ [33].

Table XII illustrates the feeder power per channel in the trunk of the ODN leaving the OLT, for both proposed OLT designs. The power limit per channel for the OLT split case a) only exceeds the nonlinear limit for 32 users with a $20 \mathrm{~dB}$ OA. In the AWG plus splitters case b), for 256 users an 8split at each AWG-port of the OLT is taken and for the rest of users a 4-split is employed; hence, the power limit is not reached without using an $\mathrm{OA}$ and for a $10 \mathrm{~dB} \mathrm{OA}$ is not exceeded for 256 and 128 users.

TABLE XII

FEEDER POWER (dBm) PER CHANNEL IN THE ODN TRUNK

\begin{tabular}{cccccc}
\hline \hline & \multicolumn{2}{c}{ OLT splitters } & \multicolumn{3}{c}{ ODN AWG\&splitters } \\
\multirow{2}{*}{ Users } & $10 \mathrm{~dB}$ & $20 \mathrm{~dB}$ & \multirow{2}{*}{ no OA } & $10 \mathrm{~dB}$ & $20 \mathrm{~dB}$ \\
& $\mathrm{OA}$ & $\mathrm{OA}$ & & OA & OA \\
\hline 32 & -9.5 & 0.5 & -10 & 0 & 10 \\
64 & -13 & -3 & -10 & 0 & 10 \\
128 & -16.5 & -6.5 & -13.5 & -3.5 & 6.5 \\
256 & -20 & -10 & -17 & -7 & 3 \\
\hline \hline
\end{tabular}

\section{WAVELength Plan AND CoeXistence With LEGACY SYSTEMS}

\section{A. COCONUT bandwidth and elements requirements}

The COCONUT PON required bandwidths expressed in channels for the different transceiver architectures and for out/indoor and indoor operation conditions were evaluated and illustrated in Tables II and III, respectively.

In order to estimate the needed spectrum for wavelength planning, and supposing we work in C/L-bands, we express the required optical bandwidths in $\mathrm{nm}$ in tables XIII and XIV for out/indoor and indoor conditions, respectively.

The COCONUT PON can be installed as the only working system in a green-field deployment, but coexistence with legacy systems must be considered in the wavelength plan if working compatibility is required.
TABLE XIII

REQUIRED BANDWIDTH (nm) UNDER OUT/INDOOR OPERATING CONDITIONS

\begin{tabular}{|c|c|c|c|}
\hline \multirow[b]{2}{*}{ Users } & \multicolumn{2}{|c|}{ homodyne } & \multirow{2}{*}{$\begin{array}{c}\text { heterodyne \& } \\
\text { homodyne paired TLs } \\
1 \text { TL \& } 2 \text { TLs, } 1 \text { band }\end{array}$} \\
\hline & $2 \mathrm{TLs}, 2$ bands & 2 TLs, 1 band & \\
\hline 32 & $2 \times 1.6$ & 3.85 & 4.1 \\
\hline 64 & $2 \times 3.85$ & 7.7 & 8.4 \\
\hline 128 & $2 \times 7.75$ & 15.2 & 17.1 \\
\hline 256 & $2 \times 15.5$ & 31.15 & 36 \\
\hline
\end{tabular}

TABLE XIV

REQUIRED BANDWIDTH $(\mathrm{nm}$ ) UNDER INDOOR OPERATING CONDITIONS

\begin{tabular}{|c|c|c|c|}
\hline \multirow[b]{2}{*}{ Users } & \multicolumn{2}{|c|}{ homodyne } & $\begin{array}{c}\text { heterodyne \& } \\
\text { homodyne paired TLs }\end{array}$ \\
\hline & $2 \mathrm{TLs}, 2$ bands & $2 \mathrm{TLs}, 1$ band & 1 TL \& 2 TLs, 1 band \\
\hline 32 & $2 \times 1.6$ & 3.45 & 3.7 \\
\hline 64 & $2 \times 3.45$ & 7.25 & 8.4 \\
\hline 128 & $2 \times 7.25$ & 15.2 & 16.6 \\
\hline 256 & $2 \times 15.5$ & 31 & 35.6 \\
\hline
\end{tabular}

Currently heritage G/EPON and XG/10GE-PON systems are the scenario for coexistence with the COCONUT proposal. New recent NG-PON2 TWDM standard and its optional PtP WDM architecture are also to be regarded. When the COCONUT deployment has concurrence with any other system, it has to be non-intrusive and must avoid any interference with the standardized systems. The elements that allow the compatibility are the Coexistence Element (CE) at the OLT output and a Wavelength Blocking Filter (WBF) in front of the ONU transceiver.

The coexistence architecture for the COCONUT proposal and the different legacy systems is depicted in Fig. 24, where the CE introducing around $1 \mathrm{~dB}$ IL is a part of the ODN as well as splitters and optical fibers, bounded by the $\mathrm{S} / \mathrm{R}$ and R/S points, while the WBF is a part of the ONU.

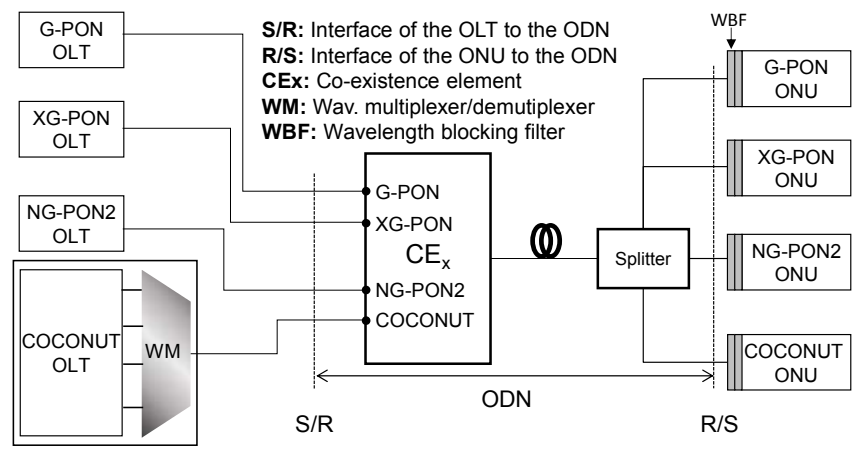

Fig. 24. Coexistence scenario: the CE allows the systems to share PON and the WBFs in the ONUs select the proper wavelengths.

When defining the wavelength plan for the COCONUT system, two issues are to be analyzed: the availability of spectrum and the quality of the deployed optical devices for compatibility. The bands defined for each standard, and the attached guard-bands, which are introduced to provide enough isolation by the optical elements to avoid crosstalk between the deployed systems, are to be taken into account. 


\section{B. Wavelength plan}

The spectrum band distribution for the different standards is depicted in Fig. 25, showing all standardized systems. In NG-PON2, the optional PtP WDM PON has two possibilities: a wide Expanded Spectrum, where particular sub-bands could be used in absence of any legacy system, or a narrow Shared Spectrum shown in Fig. 25, allowing full coexistence with all legacy systems.

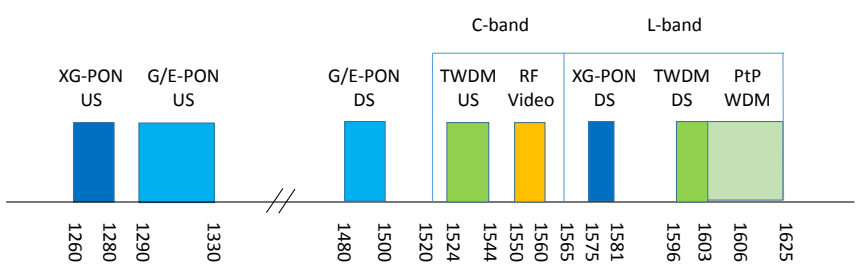

Fig. 25. Spectrum band distribution of legacy PON systems.

When the coherent detection is employed, crosstalk interaction between COCONUT and the other PON systems is reduced, especially when using PSK or FSK modulation, thus the guard bands can be narrowed [34].

A first proposal is making COCONUT compatible with all legacy systems, including NG-PON2 with PtP Shared Spectrum. In this case, only 32 or 64 users are considered, needing small bandwidths. For out/indoor conditions and with 64 users: when using 2 TLs in different bands we need 2 bands of $3.85 \mathrm{~nm}$; and a unique band of $7.7 \mathrm{~nm}$ or $8.4 \mathrm{~nm}$ for the homodyne 2 TLs or the paired lasers and heterodyne cases, respectively. These bands can be allocated in the center of the free $15 \mathrm{~nm}$ between the RF-video and the XGPON DS or between the XG-PON DS and the TWDM DS, but the WBF requirements of legacy systems could be reviewed. By deploying 32 users, the required bandwidths are minor, so they can be allocated in the same free bands.

A second proposal avoids the coexistence with NG-PON2, because if COCONUT is deployed better services are offered and there is no need to install another new system. Again for out/indoor conditions and now with 256 users: when using 2 TLs in different bands we need 2 bands of $15.5 \mathrm{~nm}$; and with 2 TLs a unique band of $31.15 \mathrm{~nm}$ or $36 \mathrm{~nm}$ for the homodyne or heterodyne instances are needed, respectively. For the first case of 2 TLs in separated bands, one $15.5 \mathrm{~nm}$ US band can be allocated before the RF-video in the same place of the TWDM US and the other $15.5 \mathrm{~nm}$ DS band in the equal place of the PtP Shared Spectrum, in that way the same CE designed for the NG-PON2 can be employed.

The required double band of $15.5 \mathrm{~nm}$ and the wider sole bands of 31.15 or $36 \mathrm{~nm}$ can be allocated after the XG-PON DS, as case (1) shown in Fig. 26, with a guard band of $9 \mathrm{~nm}$, beginning at $1589 \mathrm{~nm}$ and ending at 1620, 1620.15 or 1625 $\mathrm{nm}$ for the double band, or the sole band homodyne and heterodyne cases, respectively.

Another more practical possibility is to ignore the RFvideo band, because it has low usage, while the video is transmitted as IP-TV, as proposed case (2) shown in Fig. 26. Then, all required bands can begin at $1524 \mathrm{~nm}$ and will last at $1555,1555.15$ or $1560 \mathrm{~nm}$, for the two different bands, heterodyne and homodyne unique band cases, respectively.
If only 128 users, or even fewer such as 64 or 32 users, are deployed, the required bandwidths are even lower, so they can be allocated in the same places beginning at 1589 $\mathrm{nm}$ or at $1524 \mathrm{~nm}$ for the cases (1) or (2) respectively.

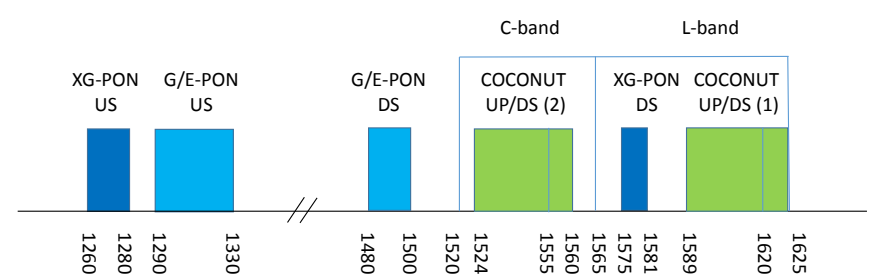

Fig. 26. Proposed spectrum band distributions for COCONUT system coexisting with G/EPON and XG-PON legacy systems: (1) in L-band or (2) in C-band without RF-video coexistence.

\section{Conclusion}

A novel UDWDM-PON with ultra-dense channel spacing has been presented. The standard passive optical tree with 32 or 64 splitting is preserved in the ODN, but it can be also augmented to 128 or 256. The new COCONUT system is based on low-cost coherent transceivers, thus avoiding optical filters and providing high sensitivity.

Several ONU architectures have been introduced: with two lasers in different bands and in the same band with homodyne or heterodyne transmission formats. At the OLT two architectures are proposed: splitter based and two thermal AWGs plus splitters; where measurements of the AWG tunability show a spectral response with low ripple.

The lasers at the ONUs are of the low-cost DFB type, with limited thermal tunability and distributed in subbands approximating a uniform wavelength distribution in a band, defining a SUDWDM approach and exploiting the wavelength-to-the-user concept. To manage the wavelength channel distribution, three heuristic DWA schemes in static and dynamic paradigms have been tested in activation processes and in operation conditions: FF, MS and MA. Simulations show that MA algorithm with dynamic assignment furnishes the lowest rejection in activation and also the minimum blocking probability in operation under environmental conditions. To enhance the assignment in harsh outdoor conditions, the original band is extended adding guard bands at both sides of the previous band, thus improving dramatically the blocking probability. Another issue is estimating the OAR for the most blocked ONU, reaching a $99.9 \%$ of availability with low operation bandwidths. The required bandwidths are evaluated for all ONU architectures and deployed number of users.

When exploiting the channels, a DWTTU is assumed instead of SWTTU, because enough bandwidth is available; therefore, each user can profit full bit rate in a channel.

The network is dimensioned for all deployed users and ONU architectures. The number of required sub-bands to furnish a uniform wavelength distribution of DFB-lasers is calculated to provision correctly the ONUs for activation processes and the OLT for operation conditions.

OLT architectures for both splitters and thermal AWGs based designs are fulfilled and implemented. The system reach, the power safety and the nonlinearities are evaluated 
and compared. For a low number of users up to 64, the split based OLT architecture by employing an OA is enough to achieve a long reach; but with a higher number of users up to 256 , the AWG based OLT architecture with a $10 \mathrm{~dB}$ OA improves the LB, while respecting the power safety and keeping the nonlinearities below the limit.

Finally, COCONUT wavelength planning and coexistence with legacy systems is envisioned: deployments of 32 or 64 users can be compatible, including NG-PON2; but installing 128 or 256 users requires higher bandwidths, and then coexistence is only projected with G/EPON and XG-PON.

\section{REFERENCES}

[1] ITU-T G.987, "10-Gigabit-capable passive optical network (XGPON) systems", Geneva, Switzerland, 2012.

[2] 10G EPON standard IEEE P802.3av, 2009.

[3] Y. C. Chung, "Recent advancement in WDM-PON technology," in Proc. ECOC, Th.11.C.4, Geneva, Switzerland, 2011.

[4] J.Prat, V. Polo, J. A. Lázaro, F. Bonada, E. Lopez, B. Schrenk, M. Omella, F. Saliou, Q. T. Le, P. Chanclou, D. Leino, R. Soila, F. Spirou, L. Costa, A. Teixeira, G.M. Tosi-Beleffi, D. Klonidis, and I. Tomkos, "Demonstration and field trial of a scalable and resilient hybrid ngPON," in Proc. ECOC, Tu.6.C.3, Geneva, Switzerland, 2011.

[5] D. Qian, N. Cvijetic, J. Hu, and T. Wang, "A novel OFDM-PON architecture with source-free ONUs for next-generation optical access networks," IEEE Photonics Technology Letters, vol. 21, no. 17 , pp. 1265-1267, 2009.

[6] D. van Veen, W. Pohlmann, J. Galaro, B. Deppisch, A. Duque, M. F. Lau, B. Farah, T. Pfeiffer, and P. Vetter, "System demonstration of a time and wavelength-set division multiplexing PON," in Proc. ECOC, We.3.F.2, London, UK, 2013.

[7] ITU-T, Recommendation G.989.2, "40-Gigabit-capable passive optical networks 2 (NGPON2): Physical media dependent (PMD) layer specification," Dec. 2014

[8] R. Murano, "Optical component technology options for NGPON2 systems," in Proc. OFC, M31.1, San Francisco, CA, USA, 2014.

[9] R. Murano, and M. J. L. Cahill, "Low cost tunable receivers for wavelength agile PONs," in Proc. ECOC, We.2B.3, Amsterdam, The Netherlands, 2012.

[10] J. M. Fàbrega, and J. Prat, "Ultra-Dense, transparent and resilient ring-tree access network using coupler-based remote nodes and homodyne transceivers," in Proc. ICTON, Th.B3.3, Ponta Delgada, Azores, Portugal, 2009.

[11] ITU-T, Recommendation G.694.1, "Spectral grids for WDM applications: DWDM frequency grid," Geneva, Switzerland, Feb. 2012.

[12] A. Teixeira, A. Shahpari, J. D. Reis, and R. Ferreira, "Flexible Access networks," in Proc. ICTON, Tu.B3.1, Graz, Austria, 2014.

[13] J. Prat, M. Angelou, C. Kazmierski, R. Pous, M. Presi, A. Rafel, G. Vall-llosera, I. Tomkos, and E. Ciaramella, "Towards ultradense wavelength to-the-user: the approach of the COCONUT project," in Proc. ICTON, Tu.C3.2, Cartagena, Spain, 2013.

[14] J. Prat, "Technologies for a cost effective UDWDM-PON," in Proc. OFC, Th3I.3, Los Angeles, CA, USA, 2015.

[15] N. Cheng, G. Wei, and F. Effenberger, "Dynamic spectrum managed passive optical networks," IEEE Communications Magazine, vol. 49, no.11, pp. 86-93, 2011.

[16] T. Garrod, F. Brunet, C. Galstad, M. Klaus, D. Olson, C. Zenner, Y. Xiao, L. Mawst, and D. Botez, "High-power and high-efficiency distributed feedback (DFB) lasers operating in the 1.4-1.6 $\mathrm{\mu m}$ range for eye-safe applications," in Proc. SPIE 8605, High-Power Diode Laser Technology and Applications XI, 86050I, S. Francisco, CA, USA, 2013.
[17] H. Rohde, S. Smolorz, E. Gottwald, and K. Kloppe, "Next generation optical access: $1 \mathrm{Gbit} / \mathrm{s}$ for everyone," in Proc. ECOC, 10.5.5, Vienna, Austria, 2009

[18] I. N. Cano, A. Lerín, V. Polo, and J. Prat, "Direct phase modulation DFBs for cost-effective ONU transmitter in udWDM PONs," IEEE Photonics Technology Letters, vol. 26, no. 10, pp. 973-975, 2014.

[19] V. Sales, J. Segarra, V. Polo, and J. Prat, "Statistical UDWDMPONs operating with ONU lasers under limited tunability," IEEE Photonics Technology Letters, vol. 27, no.3, pp. 257-260, 2015.

[20] J. Prat, V. Polo, P. Zakynthinos, I. Cano, J. A. Tabares, J. M. Fàbrega, D. Klonidis, and I. Tomkos,: "Simple intradyne PSK system for udWDM-PON," in Proc. ECOC, We.2.B.2, Amsterdam, The Netherlands, 2012.

[21] E. Ciaramella,"Polarization-independent receivers for low-cost coherent OOK systems," IEEE Photonics Technology Letters, vol. 26, no.6, pp. 548-551, 2014.

[22] I. N. Cano, A. Lerín, V. Polo, and J. Prat, "Flexible D(Q)PSK 1.25-5 Gb/s UDWDM-PON with directly modulated DFBs and centralized polarization scrambling," in Proc. ECOC, Th.1.3.7, Valencia, Spain, 2015.

[23] J. Segarra, V. Sales, V. Polo, and J. Prat, "Half-Duplex transmission avoiding Rayleigh Backscattering crosstalk in UDWDM-PON with coherent receivers," in Proc. ICTON, Mo.C3.5, Graz, Austria, 2014.

[24] J. Segarra, V. Sales, J. Prat, and R. Pous, "A new flexible ONU design for UDWDM-PON with coherent transceivers and smart activation process," in Proc. Networks, thu.s11.2, Funchal, Madeira, Portugal, 2014.

[25] G. Y. Chu, A. Lerín, I. N. Cano, V. Polo, J. A. Tabares, and J. Prat, "Exploiting RSOA for uplink transmission with coherent detection for low cost UDWDM-PON," in Proc. ACP, AF2B.1, Shanghai, China, 2014.

[26] A. Lerín, G. Y. Chu, V. Polo, I. N. Cano, V. Polo, and J. Prat, "Chip integrated DFB-EAM directly phase modulation performance improvement in UDWDM-PON," in Proc. ECOC, P.7.10, Valencia, Spain, 2015

[27] G. Y. Chu, I. N. Cano, C. Kazmierski, R. Benoit, and J. Prat, "First demonstration of monolithically integrated dual output DEML for full-duplex UDWDM-PON ONU," in Proc. ECOC, Th.1.3.1, Valencia, Spain, 2015.

[28] C. N. Ververidis, I. Tomkos, D. Klonidis, A. Rafel, N. Parkin, P. Urban, J. Prat, and J. Segarra, "Control and management requirements for a coherent ultra-dense WDM PON for lambda to the user access networks," in Proc. ICTON, Mo.C3.6, Graz, Austria, 2014.

[29] M. P. McGarry, M. Reisslein, M. Maier, and K Keha, "Bandwidth management for WDM PONs," Journal of Optical Networking, vol. 5, no. 9, pp. 637-654, 2006.

[30] I. Cano, M. C. Santos, V. Polo, and J. Prat, "Dimensioning of OFDMA PON with non-preselected-independent ONUs sources and wavelength-control," in Proc. ECOC, Tu.5.C.2, Geneva, Switzerland, 2011.

[31] ITU-T G.827, "Availability performance parameters and objectives for end-to-end international constant bit-rate digital paths," Geneva, Switzerland, 2003.

[32] IEC 60825-2, "Safety of laser products - Part 2: Safety of optical fibre communication systems (OFCS)," 3rd ed., Geneva, Switzerland, 2004.

[33] H. Rohde, E. Gottwall, A. Teixeira, J. Dias Reis, A. Shahpari, K. Pulverer, and J. Shan Wey, "Coherent ultra dense WDM technology for next generation optical metro and access networks," IEEE Journal of Lightwave Technology, vol. 32, no. 10, pp. 2041-2052, 2014.

[34] J. Prat, I. Cano, M. Presi, I Tomkos, D. Klonidis, G. Vallllosera, R. Brenot, R. Pous, G. Papastergiou, A. Rafel and E. Ciaramella, "Technologies for cost effective udWDM-PONs," IEEE Journal of Lightwave Technology, vol. 34, no. 2, pp. 783791, 2016. 